\title{
A phase-field model with convection: sharp-interface asymptotics
}

\author{
D.M. Anderson ${ }^{\mathrm{a}, *}$, G.B. McFadden ${ }^{\mathrm{b}}$, A.A. Wheeler ${ }^{\mathrm{c}}$ \\ a Department of Mathematical Sciences, George Mason University, Fairfax, VA 22030, USA \\ b Mathematical and Computational Sciences Division, National Institute of Standards and Technology, Gaithersburg, MD 20899-8910, USA \\ ${ }^{\mathrm{c}}$ Faculty of Mathematical Studies, University of Southampton, Highfield, Southampton SO17 1BJ, UK \\ Received 2 October 2000; received in revised form 19 January 2001; accepted 29 January 2001 \\ Communicated by C.K.R.T. Jones
}

\begin{abstract}
We have previously developed a phase-field model of solidification that includes convection in the melt [Physica D 135 (2000) 175]. This model represents the two phases as viscous liquids, where the putative solid phase has a viscosity much larger than the liquid phase. The object of this paper is to examine in detail a simplified version of the governing equations for this phase-field model in the sharp-interface limit to derive the interfacial conditions of the associated free-boundary problem. The importance of this analysis is that it reveals the underlying physical mechanisms built into the phase-field model in the context of a free-boundary problem and, in turn, provides a further validation of the model. In equilibrium, we recover the standard interfacial conditions including the Young-Laplace and Clausius-Clapeyron equations that relate the temperature to the pressures in the two bulk phases, the interface curvature and material parameters. In nonequilibrium, we identify boundary conditions associated with classical hydrodynamics, such as the normal mass flux condition, the no-slip condition and stress balances. We also identify the heat flux balance condition which is modified to account for the flow, interface curvature and density difference between the bulk phases. The interface temperature satisfies a nonequilibrium version of the Clausius-Clapeyron relation which includes the effects of curvature, attachment kinetics and viscous dissipation. (C) 2001 Published by Elsevier Science B.V.
\end{abstract}

PACS: 05.70.Fh; 05.70.Ln; 81.10.Fq

Keywords: Phase-field; Convection; Solidification; Sharp-interface analysis

\section{Introduction}

In the recent paper [1] (AMW), we derived a phase-field model that describes the solidification of a pure material when fluid motion is present. The motivation for developing and studying such a model follows from experimental observations and theoretical analyses which show that fluid motion in the melt can have a profound effect on the morphology of the solid-liquid interface [2-6]. In an industrial setting morphological and hydrodynamic instabilities can have a detrimental effect on both the control of the process and the quality of the solidified product. However, even when careful attempts are made to suppress fluid motion by eliminating external forces such as gravity, it

\footnotetext{
* Corresponding author.

E-mail address: danders1@gmu.edu (D.M. Anderson).
} 
is not always possible to eliminate all fluid motion. For example, expansion or contraction flow is present during solidification when the solid and liquid densities of the material are different. Furthermore, even in equilibrium where fluid motion is absent, the presence of a density difference between the solid and liquid phases can have thermodynamic consequences.

Typical phase-field models for a pure material without convection [7-10] introduce a phase-field variable $\phi(\vec{x}, t)$, which is a function of position $\vec{x}$ and time $t$, to distinguish the solid from the liquid. This is in contrast to diffuse interface descriptions of a fluid near its critical point, where density is a more natural order parameter [11,12]. The phase-field governing equations have been derived in a thermodynamically consistent way by Penrose and Fife and coworkers [10,13,14] following the formalism of irreversible thermodynamics [15]. AMW followed these same principles to derive their phase-field model that includes convection. They retained the use of the phase-field variable $\phi$ and allowed the density of each phase to be specified independently. The model was developed to describe fluid motion that is incompressible in the bulk phases. Therefore, in order to account for expansion or contraction flows that are driven from solidification at the solid-liquid interface they incorporated the ideas of quasi-incompressibility first used in a diffuse-interface description of a binary fluid by Lowengrub and Truskinovsky [16].

AMW treated both phases as fluids, although they examined the case where one phase has a sufficiently large viscosity, so that it may be interpreted as an approximation to a solid phase. In many solidification applications, a fluid model is used for the thermodynamic description of the solid phase, in that the elastic properties of the solid are ignored. AMW also assumed that the phase transition was first-order and had an anisotropic surface energy, which is unconventional for a fluid-fluid system, but is consistent with our primary intention of modeling a solid-liquid system.

There have been a number of other phase-field type descriptions of solidification that include convection in the melt [17-21]. The AMW model (see also Ref. [22]) incorporates in a thermodynamically consistent way the density effect in a phase-field description of solidification as well as the appropriate form of the Korteweg stress term in the momentum equation.

The object of this paper is to examine in detail, the governing equations for the phase-field convection model of AMW (see also Ref. [22]) in the sharp-interface limit to derive the interfacial conditions of the associated free-boundary problem. The importance of this analysis is that it reveals the underlying physical mechanisms built into the phase-field model in the context of a free-boundary problem and, in turn, provides a further validation of the model. To date sharp-interface analyses of phase-field models have concentrated on situations in which flow is not present and the densities of both phases are the same [23-30]. The version of the AMW model without fluid flow has recently been examined in detail [30]. Therefore, the focus in the present work is on the effects associated with density differences between the bulk phases as well as fluid flow and how they manifest themselves in the sharp-interface limit. We follow the so-called "classical" sharp-interface analysis and refer the reader to [26-30] for other possible distinguished limits and related issues.

The paper is organized as follows. The governing equations are described briefly in Section 2 and their dimensionless forms used in the present analysis are given in Section 3. The sharp-interface analysis is first examined for the equilibrium case in Section 4. Section 5 discusses scaling issues associated with the nonequilibrium version of the equations which are then analyzed in two different limits. Conclusions are given in Section 6.

\section{Governing equations}

In the absence of convection, phase-field models can be derived by extending the formalism of classical irreversible thermodynamics [15] to nonclassical systems whose thermodynamic potentials involve square-gradient energy terms $[10,13,14]$. AMW built on these ideas to develop a phase-field model that incorporates convection. The governing 
equations of this model which describe convection, heat transfer and solidification of a pure material are

$$
\begin{aligned}
& \frac{\mathrm{D} \rho}{\mathrm{D} t}=-\rho \nabla \cdot \vec{u}, \\
& \rho \frac{\mathrm{D} \vec{u}}{\mathrm{D} t}=\nabla \cdot \boldsymbol{m}, \\
& M \frac{\mathrm{D} \phi}{\mathrm{D} t}=\epsilon_{F}^{2}(T) \nabla \cdot[\Gamma(\nabla \phi) \vec{\xi}]-\rho \frac{\partial g}{\partial \phi}, \\
& \rho \frac{\mathrm{D} e}{\mathrm{D} t}=\nabla \cdot[k(\phi) \nabla T]+\epsilon_{E}^{2} \nabla \cdot[\Gamma(\nabla \phi) \vec{\xi}] \frac{\mathrm{D} \phi}{\mathrm{D} t}+\boldsymbol{m}_{S}: \nabla \vec{u},
\end{aligned}
$$

where $\rho$ is the density, $\vec{u}$ the velocity, $\boldsymbol{m}$ the stress tensor, $\phi$ the phase-field variable (we adopt the convention that $\phi=0$ denotes the liquid phase and $\phi=1$ denotes the solid phase), and $T$ the temperature. Additionally, $M$ is a constant that represents the mobility of the interface, and $\epsilon_{F}$ the Helmholtz gradient energy coefficient given by $\epsilon_{F}^{2}(T)=\epsilon_{E}^{2}+T \epsilon_{S}^{2}$, where $\epsilon_{E}$ and $\epsilon_{S}$ are the gradient energy and entropy coefficients which are assumed to be constants. Further, $k(\phi)$ is the thermal conductivity, and $g(T, p, \phi)=e-T s+p / \rho$ is the Gibbs free energy per unit mass, where $e$ is the internal energy per unit mass, $s$ the entropy per unit mass and $p$ the pressure. The quantities $\boldsymbol{m}$ and $\boldsymbol{m}_{S}$ are given by

$$
\begin{aligned}
& \boldsymbol{m}=\left[-p+\frac{1}{2} \epsilon_{F}^{2}(T) \Gamma^{2}(\nabla \phi)\right] \boldsymbol{I}-\epsilon_{F}^{2}(T) \Gamma(\nabla \phi) \vec{\xi} \otimes \nabla \phi+\boldsymbol{\tau}, \\
& \boldsymbol{m}_{S}=\left[-p+\frac{1}{2} T \epsilon_{S}^{2} \Gamma^{2}(\nabla \phi)\right] \boldsymbol{I}-T \epsilon_{S}^{2} \Gamma(\nabla \phi) \vec{\xi} \otimes \nabla \phi+\boldsymbol{\tau},
\end{aligned}
$$

where $\tau$ is the viscous stress tensor

$$
\boldsymbol{\tau}=\mu(\phi)\left[\nabla \vec{u}+(\nabla \vec{u})^{\mathrm{T}}-\frac{2}{3}(\nabla \cdot \vec{u}) \boldsymbol{I}\right],
$$

and $\mu(\phi)$ is the viscosity which is a function of $\phi$ given by

$$
\mu(\phi)=\mu_{\mathrm{S}} r(\phi)+\mu_{\mathrm{L}}[1-r(\phi)],
$$

where $\mu_{\mathrm{S}}$ and $\mu_{\mathrm{L}}$ are the viscosities in the bulk solid and liquid phases, respectively. The function $r(\phi)$ is monotonic increasing with $r(0)=0$ and $r(1)=1$; suitable choices include $r(\phi)=\phi$ or $r(\phi)=\phi^{2}(3-2 \phi) . \Gamma(\nabla \phi)$ is a homogeneous function of degree unity that permits a general anisotropic surface energy of the solid-liquid interface, and $\vec{\xi}$ is the generalized $\xi$-vector whose components are defined by $\xi_{j}=\partial \Gamma(\nabla \phi) / \partial \phi, j$, where $\phi_{, j}$ is the $j$ th component of $\nabla \phi$, see [31,32]. We will henceforth confine our attention to case of isotropic surface energies and set $\Gamma(\nabla \phi)=|\nabla \phi|$, in which case $\vec{\xi}=\nabla \phi /|\nabla \phi|$.

The density of the two bulk phases may be different, and we shall assume that $\rho$ depends on $\phi$ alone,

$$
\rho(\phi)=\rho_{\mathrm{S}} r(\phi)+\rho_{\mathrm{L}}[1-r(\phi)]
$$

where $\rho_{\mathrm{S}}$ and $\rho_{\mathrm{L}}$ are the densities in the bulk solid and liquid phases, respectively. This assumption, in which the density does not depend on temperature or pressure, known as quasi-incompressibility, places a constraint on the form of the underlying thermodynamic potentials [16] and requires that the underlying Gibbs free energy density be given by

$$
g(T, p, \phi)=g_{0}(T, \phi)+\frac{p-p_{\mathrm{R}}}{\rho(\phi)},
$$


where $p_{\mathrm{R}}$ is a reference pressure associated with an isothermal stationary planar interface at melting temperature $T_{\mathrm{M}}$. We take $g_{0}(T, \phi)$ to have the form

$$
g_{0}(T, \phi)=\left[e_{0}-c T_{\mathrm{M}}-r(\phi) L-\frac{1}{4 a_{S}} H_{\mathrm{m}}(\phi)\right]\left(1-\frac{T}{T_{\mathrm{M}}}\right)-c T \ln \left(\frac{T}{T_{\mathrm{M}}}\right)+\frac{1}{4 a} H_{\mathrm{m}}(\phi),
$$

in which case the corresponding expressions for the internal energy and entropy densities are

$$
\begin{aligned}
& e=e_{0}+c\left(T-T_{\mathrm{M}}\right)-r(\phi) L+\frac{1}{4 a_{E}} H_{\mathrm{m}}(\phi)-\frac{p_{\mathrm{R}}}{\rho(\phi)}, \\
& s=\frac{1}{T_{\mathrm{M}}}\left[e_{0}-r(\phi) L-\frac{1}{4 a_{S}} H_{\mathrm{m}}(\phi)\right]+c \ln \left(\frac{T}{T_{\mathrm{M}}}\right),
\end{aligned}
$$

where $1 / a_{E}=1 / a-1 / a_{S}$. Here $1 / a$ is the height of the double-well of the Gibbs free energy density at $T=T_{\mathrm{M}}$, and $1 / a_{E}$ and $1 / a_{S}$ are the heights of the double wells in the internal energy and entropy densities, respectively. The quantity $e_{0}$ is a constant reference energy and both the heat capacity per unit mass $c$ and the latent heat per unit mass $L$ are assumed to be constant.

The double-well function $H_{\mathrm{m}}(\phi)$ appears in the "per unit mass" quantity $g_{0}(T, \phi)$. The analog "per unit volume" quantity associated with $\rho(\phi) g_{0}(T, \phi)$ is $\rho(\phi) H_{\mathrm{m}}(\phi) \equiv H_{\mathrm{v}}(\phi)$. A common form of a double-well potential is the function $\phi^{2}(1-\phi)^{2}$, and in standard phase-field models that do not include convection it is usually associated with a "per unit volume" quantity (e.g. [10]). In applications, in which the density of the two phases are constant and equal, the per unit mass and the per unit volume specification of the double-well are equivalent. However, in the present situation the bulk densities are not necessarily equal and therefore we shall examine the merits of the per unit mass form $H_{\mathrm{m}}(\phi)=\phi^{2}(1-\phi)^{2}$ and the per unit volume form $H_{\mathrm{v}}(\phi)=\rho_{\mathrm{L}} \phi^{2}(1-\phi)^{2}$ (in which case $\left.H_{\mathrm{m}}(\phi)=\rho_{\mathrm{L}} \phi^{2}(1-\phi)^{2} / \rho(\phi)\right)$.

The one-dimensional solution of the governing equations for an isothermal system at the melting temperature $T_{\mathrm{M}}$ with equal solid and liquid densities $\left(\rho_{\mathrm{S}}=\rho_{\mathrm{L}}\right)$ is given by

$$
\begin{aligned}
& \phi=\frac{1}{2}\left[1-\tanh \left(\frac{z}{2 l}\right)\right], \\
& p=p_{\mathrm{R}}-\frac{3 \gamma_{0}}{16 l} \operatorname{sech}^{4}\left(\frac{z}{2 l}\right) .
\end{aligned}
$$

The interface thickness, $l$, and surface energy $\gamma_{0}$, are related to the phase-field parameters by

$$
l=\epsilon_{F}\left(T_{\mathrm{M}}\right) \sqrt{\frac{2 a}{\rho_{\mathrm{L}}}}, \quad \gamma_{0}=\frac{\epsilon_{F}\left(T_{\mathrm{M}}\right)}{6} \sqrt{\frac{\rho_{\mathrm{L}}}{2 a}} .
$$

It is also convenient to define an associated capillary length by $l_{\mathrm{c}}=T_{\mathrm{M}} \gamma_{0} /\left(\rho_{\mathrm{L}}\left[L^{2} / c\right]\right)$.

\section{Dimensionless governing equations}

We nondimensionalise the governing equations by introducing a reference length scale $l_{0}$, which is a typical length scale associated with the interface shape, such as a dendrite tip radius. The reference scales for time, velocity, pressure and stress are based on thermal diffusion in the liquid. The temperature scale is $L / c$ and the density, viscosity and thermal conductivity are scaled with their values in the bulk liquid. The dimensionless quantities, 
denoted by primes, are related to their dimensional counterparts by

$$
\begin{aligned}
& \vec{x}=l_{0} \vec{x}^{\prime}, \quad t=\frac{l_{0}^{2}}{\kappa_{\mathrm{L}}} t^{\prime}, \quad \vec{u}=\frac{\kappa_{\mathrm{L}}}{l_{0}} \vec{u}^{\prime}, \quad \boldsymbol{m}=\frac{\rho_{\mathrm{L}} \kappa_{\mathrm{L}}^{2}}{l_{0}^{2}} \boldsymbol{m}^{\prime}, \quad p=p_{\mathrm{R}}+\frac{\rho_{\mathrm{L}} \kappa_{\mathrm{L}}^{2}}{l_{0}^{2}} p^{\prime}, \\
& T=T_{\mathrm{M}}+\frac{L}{c} \theta^{\prime}, \quad \rho=\rho_{\mathrm{L}} \rho^{\prime}, \quad \mu=\mu_{\mathrm{L}} \mu^{\prime}, \quad k=k_{\mathrm{L}} k^{\prime},
\end{aligned}
$$

where $\kappa_{\mathrm{L}}$ is the thermal diffusivity in the bulk liquid phase. The dimensionless governing equations are

$$
\begin{aligned}
& \frac{\partial \rho}{\partial t}+\nabla \cdot(\rho \vec{u})=0, \\
& \rho \frac{\mathrm{D} \vec{u}}{\mathrm{D} t}=\nabla \cdot \boldsymbol{m}, \\
& \epsilon^{2} \tau \frac{\mathrm{D} \phi}{\mathrm{D} t}=\epsilon^{2}(1+\alpha \theta) \nabla^{2} \phi-\rho\left[\frac{1}{2}(1+\beta \theta) H_{\mathrm{m}}^{\prime}(\phi)+\lambda \theta r^{\prime}(\phi)+\frac{\epsilon}{\tilde{\gamma}} p \frac{\partial}{\partial \phi}\left(\frac{1}{\rho}\right)\right], \\
& \rho \frac{\mathrm{D} e}{\mathrm{D} t}=\nabla \cdot[Q(\phi) \nabla \theta]+\epsilon^{2} \nu \frac{\mathrm{D} \phi}{\mathrm{D} t} \nabla^{2} \phi+\mathcal{H},
\end{aligned}
$$

where, for simplicity, we have omitted the primes on the dimensionless variables. The dimensionless internal energy density, entropy and Gibbs free energy are given by

$$
\begin{aligned}
& e=\theta-r(\phi)+\frac{\delta}{2} H_{\mathrm{m}}(\phi)-\frac{\epsilon S p^{\star}}{\lambda \tilde{\gamma}} \frac{1}{\rho}, \\
& s=\lambda e_{0}^{\prime}-\lambda r(\phi)-\frac{1}{2} \beta H_{\mathrm{m}}(\phi)+\frac{\lambda}{S} \ln (1+S \theta), \\
& g=-\lambda\left(e_{0}^{\prime}-S^{-1}\right) \theta-\frac{\lambda}{S^{2}}(1+S \theta) \ln (1+S \theta)+\frac{1}{2} H_{\mathrm{m}}(\phi)+\lambda \theta r(\phi)+\frac{\epsilon}{\tilde{\gamma}} \frac{p}{\rho(\phi)},
\end{aligned}
$$

where $e_{0}^{\prime}=e_{0} / L$. Note that $g$ is scaled with $1 /(2 a), s$ with $c /(2 a L)$ and $e-e_{0}$ with $L$. The dimensionless tensors are

$$
\begin{aligned}
& \boldsymbol{m}=\boldsymbol{\sigma}^{p}+(1+\alpha \theta) \boldsymbol{\sigma}^{\phi}+\operatorname{Pr} \boldsymbol{\tau}, \\
& \boldsymbol{m}_{S}=\boldsymbol{\sigma}^{p}+\alpha\left(\theta+S^{-1}\right) \boldsymbol{\sigma}^{\phi}+\operatorname{Pr} \boldsymbol{\tau},
\end{aligned}
$$

where

$$
\begin{aligned}
& \boldsymbol{\sigma}^{p}=-\left(p+p^{\star}\right) \boldsymbol{I}, \\
& \boldsymbol{\sigma}^{\phi}=\tilde{\gamma} \epsilon\left[\frac{1}{2}|\nabla \phi|^{2} \boldsymbol{I}-\nabla \phi \otimes \nabla \phi\right], \\
& \boldsymbol{\tau}=\mu(\phi)\left[\nabla \vec{u}+(\nabla \vec{u})^{\mathrm{T}}-\frac{2}{3}(\nabla \cdot \vec{u}) \boldsymbol{I}\right] .
\end{aligned}
$$

The source term in the energy equation is

$$
\mathcal{H}=\frac{\epsilon S}{\lambda \tilde{\gamma}} \boldsymbol{m}_{S}: \nabla \vec{u}=\frac{\epsilon S}{\lambda \tilde{\gamma}}\left[-\left(p+p^{\star}\right) \boldsymbol{I}+\alpha\left(\theta+S^{-1}\right) \boldsymbol{\sigma}^{\phi}+\operatorname{Pr} \boldsymbol{\tau}\right]: \nabla \vec{u} .
$$

The dimensionless parameters are given by

$$
\alpha=\frac{L \epsilon_{S}^{2}}{c \epsilon_{F}^{2}\left(T_{\mathrm{M}}\right)}, \quad \beta=\frac{a L}{a_{S} c T_{\mathrm{M}}}, \quad \delta=\frac{1}{2 a_{E} L}, \quad v=\frac{\epsilon_{E}^{2}}{l^{2} L \rho_{\mathrm{L}}}=\frac{\epsilon_{E}^{2}}{2 a L \epsilon_{F}^{2}\left(T_{\mathrm{M}}\right)},
$$




$$
\begin{array}{ll}
\epsilon=\frac{l}{l_{0}}, \quad \lambda=\frac{l}{6 l_{\mathrm{c}}}, \quad p^{\star}=\frac{p_{\mathrm{R}} l_{0}^{2}}{\rho_{\mathrm{L}} \kappa_{\mathrm{L}}^{2}}, \quad S=\frac{L}{c T_{\mathrm{M}}}, \quad \operatorname{Pr}=\frac{\nu_{\mathrm{L}}}{\kappa_{\mathrm{L}}}, \\
\tilde{\gamma}=\frac{6 l_{0} l_{\mathrm{c}} L^{2}}{\kappa_{\mathrm{L}}^{2} c T_{\mathrm{M}}}=\frac{6 l_{0} \gamma_{0}}{\rho_{\mathrm{L}} \kappa_{\mathrm{L}}^{2}}, \quad \tau=\frac{\kappa_{\mathrm{L}} M T_{\mathrm{M}} c}{6 \rho_{\mathrm{L}} l l_{\mathrm{c}} L^{2}}=\frac{\left[\kappa_{\mathrm{L}} / l_{\mathrm{c}}\right]}{[L / c] \mu_{\mathrm{mob}}},
\end{array}
$$

where $v_{\mathrm{L}}$ is the kinematic viscosity of the liquid phase and

$$
Q(\phi)=1+\left(\frac{k_{\mathrm{S}}}{k_{\mathrm{L}}}-1\right) r(\phi), \quad \mu(\phi)=1+\left(\frac{\mu_{\mathrm{S}}}{\mu_{\mathrm{L}}}-1\right) r(\phi), \quad \rho(\phi)=1+\left(\frac{\rho_{\mathrm{S}}}{\rho_{\mathrm{L}}}-1\right) r(\phi) .
$$

We note that the parameter $\tilde{\gamma}$ is related to the capillary (or crispation) number $\mathrm{Ca}$ by $\tilde{\gamma}=6 \mathrm{Pr} / \mathrm{Ca}$, where $\mathrm{Ca}=$ $\mu_{\mathrm{L}} \kappa_{\mathrm{L}} / l_{0} \gamma_{0}$. Additionally, if one follows the thermodynamic relationships between the gradient coefficients and the double-well heights, namely, $\epsilon_{F}^{2}\left(T_{\mathrm{M}}\right)=\epsilon_{E}^{2}+T_{\mathrm{M}} \epsilon_{S}^{2}$ and $1 / a_{E}=(1 / a)-\left(1 / a_{S}\right)$, we find, as in [30] that $\alpha, \beta, \delta$ and $v$ are not independent parameters but rather are related through

$$
\begin{aligned}
& \beta-\alpha=\lambda(\nu-\delta), \\
& \alpha=S-\lambda \nu .
\end{aligned}
$$

In the absence of flow these equations reduce to the generalized phase-field equations recently studied in Refs. $[30,33]$. We observe that $\epsilon$ is the ratio of the interface thickness $l$ to the reference length scale $l_{0}$ and in the analysis that follows we shall consider the sharp-interface limit $\epsilon \rightarrow 0$. The leading-order free-boundary problem that emerges from a sharp-interface limit of these equations depends on the distinguished limit that is taken $[23,27,30]$. The particular sharp-interface limit in which $\lambda=\mathcal{O}(1)$ as $\epsilon \rightarrow 0$, where the interface thickness is comparable to the capillary length, is the so-called 'thin interface' limit studied by Karma and Rappel [27]. The case $\lambda=\mathcal{O}(\epsilon)$ as $\epsilon \rightarrow 0$, where the interface thickness is much smaller than the capillary length, is the so-called 'classical' limit.

In this paper, we consider a classical sharp-interface limit for the simplified model where $\alpha=\beta=0$ and $v=\delta=S / \lambda$. Here the square-gradient and double-well terms are present in the internal energy functional but not in the entropy functional. Notice that since $\lambda=\mathcal{O}(\epsilon)$ in the classical limit, we have $v=\delta=S / \lambda=\mathcal{O}\left(\epsilon^{-1}\right)$; these terms all appear in the energy equation. We refer the reader to [30], where the more general case of nonzero $\alpha, \beta$, $\delta$ and $v$ is examined without fluid flow. The dimensionless governing equations in this case are

$$
\begin{aligned}
& \frac{\partial \rho}{\partial t}+\nabla \cdot(\rho \vec{u})=0, \\
& \rho \frac{\mathrm{D} \vec{u}}{\mathrm{D} t}=\nabla \cdot \boldsymbol{m}, \\
& \epsilon^{2} \tau \frac{\mathrm{D} \phi}{\mathrm{D} t}=\epsilon^{2} \nabla^{2} \phi-\rho\left[\frac{1}{2} H_{\mathrm{m}}^{\prime}(\phi)+\lambda \theta r^{\prime}(\phi)+\frac{\epsilon}{\tilde{\gamma}} p \frac{\partial}{\partial \phi}\left(\frac{1}{\rho}\right)\right], \\
& \rho \frac{\mathrm{D} e}{\mathrm{D} t}=\nabla \cdot[Q(\phi) \nabla \theta]+\epsilon^{2} \nu \frac{\mathrm{D} \phi}{\mathrm{D} t} \nabla^{2} \phi+\mathcal{H},
\end{aligned}
$$

where $\boldsymbol{m}$ and $\mathcal{H}$ are given by Eqs. (21a) and (23) with $\alpha=0$.

\section{Equilibrium: sharp-interface analysis}

In this section, we conduct a classical sharp-interface analysis of the equilibrium version of Eqs. (29)-(32). Here the fluid velocity $\vec{u}$ and all time derivatives are zero. Additionally, the energy equation admits an isothermal solution 
where the temperature is denoted by $\theta$. In the classical limit, $\epsilon \rightarrow 0$ with $\lambda=\epsilon \Lambda$ and $\Lambda=\mathcal{O}(1)$, thin interfacial regions of thickness $\mathcal{O}(\epsilon)$ develop in $\phi$. Away from these regions the system is in the solid or liquid bulk phase and we express the solution in these so-called outer regions as regular perturbation series in powers of $\epsilon$. We write

$$
\begin{aligned}
& p\left(\zeta^{1}, \zeta^{2}, \zeta^{3}\right)=p_{0}\left(\zeta^{1}, \zeta^{2}, \zeta^{3}\right)+\epsilon p_{1}\left(\zeta^{1}, \zeta^{2}, \zeta^{3}\right)+\cdots, \\
& \phi\left(\zeta^{1}, \zeta^{2}, \zeta^{3}\right)=\phi_{0}\left(\zeta^{1}, \zeta^{2}, \zeta^{3}\right)+\epsilon \phi_{1}\left(\zeta^{1}, \zeta^{2}, \zeta^{3}\right)+\cdots,
\end{aligned}
$$

where $\zeta^{1}, \zeta^{2}, \zeta^{3}$ are coordinates locally body-fitted to the mean position of the interface, given by the surface $\phi=\frac{1}{2}$. Here $\zeta^{1}, \zeta^{2}$ are surface coordinates measuring distance along the principal directions, and $\zeta^{3}$ is a coordinate measuring distance in the direction normal to the surface $\phi=\frac{1}{2}$, which may also be expressed by $\zeta^{3}=0$. We adopt the convention that $\zeta^{3}$ is positive in the liquid and negative in the solid. Further details of the differential geometry of body-fitted coordinates are given in Appendix A.

The phase-field equation in the outer regions gives that $\phi_{0}=0$ in the liquid and $\phi_{0}=1$ in the solid. The momentum equation $\nabla \cdot \boldsymbol{m}=0$ in the outer region requires that $p_{0}\left(\zeta^{1}, \zeta^{2}, \zeta^{3}\right)$ is constant in each phase. We put

$$
p_{0}^{\mathrm{S}}=p_{\mathrm{S}}^{\star}-p^{\star}, \quad p_{0}^{\mathrm{L}}=p_{\mathrm{L}}^{\star}-p^{\star},
$$

where $p_{\mathrm{S}}^{\star}=p_{\mathrm{S}} l_{0}^{2} /\left(\rho_{\mathrm{L}} \kappa_{\mathrm{L}}^{2}\right), p_{\mathrm{L}}^{\star}=p_{\mathrm{L}} l_{0}^{2} /\left(\rho_{\mathrm{L}} \kappa_{\mathrm{L}}^{2}\right)$, and $p_{\mathrm{S}}$ and $p_{\mathrm{L}}$ are the dimensional pressures in the bulk solid and liquid phases, respectively.

We now consider the solution in the interfacial layer. We observe from the one-dimensional solution (10) that the dimensional pressure in the interface is characterized by $\gamma_{0} / l$, and hence the dimensionless pressure in the interface scales as $\left(\gamma_{0} / l\right) /\left(\rho_{\mathrm{L}} \kappa_{\mathrm{L}}^{2} / l_{0}^{2}\right)=\tilde{\gamma} /(6 \epsilon)$, where $\tilde{\gamma}=\mathcal{O}(1)$. Consequently, we write the inner expansions as

$$
\begin{aligned}
& P\left(\zeta^{1}, \zeta^{2}, \zeta\right)=\frac{1}{\epsilon}\left[P_{0}\left(\zeta^{1}, \zeta^{2}, \zeta\right)+\epsilon P_{1}\left(\zeta^{1}, \zeta^{2}, \zeta\right)+\cdots\right] \\
& \Phi\left(\zeta^{1}, \zeta^{2}, \zeta\right)=\Phi_{0}\left(\zeta^{1}, \zeta^{2}, \zeta\right)+\epsilon \Phi_{1}\left(\zeta^{1}, \zeta^{2}, \zeta\right)+\cdots
\end{aligned}
$$

Here and below we use capital letters to denote the inner variables and $\zeta=\zeta^{3} / \epsilon$ is the normal coordinate scaled to the thickness of the interfacial layer. Equilibrium conditions follow from the momentum and phase-field equations as shown below.

\subsection{Momentum equation}

We examine the first two orders of the momentum equation $\mathcal{O}\left(\epsilon^{-1}\right)$ and $\mathcal{O}(1)$ to find that

$$
\begin{aligned}
& P_{0}=c_{0}\left(\zeta^{1}, \zeta^{2}\right)-\frac{1}{2} \tilde{\gamma}\left(\frac{\partial \Phi_{0}}{\partial \zeta}\right)^{2}, \\
& P_{1}=c_{1}\left(\zeta^{1}, \zeta^{2}\right)-p^{\star}+\tilde{\gamma}\left(\mathcal{K}_{1}+\mathcal{K}_{2}\right) \int_{-\infty}^{\zeta}\left(\frac{\partial \Phi_{0}}{\partial \zeta}\right)^{2} \mathrm{~d} \zeta-\tilde{\gamma} \frac{\partial \Phi_{0}}{\partial \zeta} \frac{\partial \Phi_{1}}{\partial \zeta},
\end{aligned}
$$

where $c_{0}$ and $c_{1}$ are independent of $\zeta$.

Matching the inner and outer pressures requires

$$
0=\lim _{\zeta \rightarrow \pm \infty}\left\{p_{0}\left(\vec{r}_{0}\right)-\frac{1}{\epsilon}\left(P_{0}+\epsilon P_{1}\right)+\cdots\right\},
$$


and gives $c_{0}\left(\zeta^{1}, \zeta^{2}\right)=0$ and $c_{1}\left(\zeta^{1}, \zeta^{2}\right)=p_{\mathrm{S}}^{\star}$. Additionally,

$$
\left.p_{0}\right|_{\mathrm{S}} ^{\mathrm{L}}=\tilde{\gamma}\left(\mathcal{K}_{1}+\mathcal{K}_{2}\right) \int_{-\infty}^{+\infty}\left(\frac{\partial \Phi_{0}}{\partial \zeta}\right)^{2} \mathrm{~d} \zeta
$$

which relates the jump in the outer pressure across the interface to the interface curvature. We identify the integral factor below after treating the phase-field equation.

\subsection{Phase-field equation}

The $\mathcal{O}(1)$ inner problem for the phase-field equation can be written as

$$
0=\frac{\partial^{2} \Phi_{0}}{\partial \zeta^{2}}+\frac{1}{2} \frac{\partial}{\partial \phi}\left[\ln \left(\frac{1}{\rho\left(\Phi_{0}\right)}\right)\right]\left(\frac{\partial \Phi_{0}}{\partial \zeta}\right)^{2}-\frac{1}{2} \rho\left(\Phi_{0}\right) H_{\mathrm{m}}^{\prime}\left(\Phi_{0}\right)
$$

where we have made use of the expression (36a) for $P_{0}$. This equation can be simplified if we first multiply through by the integrating factor $\left(1 / \rho\left(\Phi_{0}\right)\right)^{1 / 2}$ to obtain

$$
0=\frac{\partial}{\partial \zeta}\left[\left(\frac{1}{\rho\left(\Phi_{0}\right)}\right)^{1 / 2} \frac{\partial \Phi_{0}}{\partial \zeta}\right]-\left(\frac{1}{\rho\left(\Phi_{0}\right)}\right)^{1 / 2} \frac{\rho\left(\Phi_{0}\right) H_{\mathrm{m}}^{\prime}\left(\Phi_{0}\right)}{2}
$$

and then multiply this result by $\left(1 / \rho\left(\Phi_{0}\right)\right)^{1 / 2} \partial \Phi_{0} / \partial \zeta$. Integration then gives the first integral

$$
0=\left(\frac{\partial \Phi_{0}}{\partial \zeta}\right)^{2}-\rho\left(\Phi_{0}\right) H_{\mathrm{m}}\left(\Phi_{0}\right)
$$

The $\mathcal{O}(\epsilon)$ inner phase-field problem can be expressed as

$$
\frac{\partial^{2} \Phi_{1}}{\partial \zeta^{2}}+\frac{\partial}{\partial \phi}\left[\ln \left(\frac{1}{\rho\left(\Phi_{0}\right)}\right)\right] \frac{\partial \Phi_{0}}{\partial \zeta} \frac{\partial \Phi_{1}}{\partial \zeta}-\left\{\frac{1}{2} \frac{\partial}{\partial \phi}\left[\rho\left(\Phi_{0}\right) H_{\mathrm{m}}^{\prime}\left(\Phi_{0}\right)\right]+\frac{\partial^{2}}{\partial \phi^{2}}\left[\ln \left(\frac{1}{\rho\left(\Phi_{0}\right)}\right)\right] \frac{P_{0}}{\tilde{\gamma}}\right\} \Phi_{1}=R_{1},
$$

where

$$
\begin{aligned}
R_{1}= & \left(\mathcal{K}_{1}+\mathcal{K}_{2}\right) \frac{\partial \Phi_{0}}{\partial \zeta}+\Lambda \theta \rho\left(\Phi_{0}\right) r^{\prime}\left(\Phi_{0}\right)+\frac{\left(p_{\mathrm{S}}^{\star}-p^{\star}\right)}{\tilde{\gamma}} \frac{\partial}{\partial \phi}\left[\ln \left(\frac{1}{\rho\left(\Phi_{0}\right)}\right)\right] \\
& +\left(\mathcal{K}_{1}+\mathcal{K}_{2}\right) \frac{\partial}{\partial \phi}\left[\ln \left(\frac{1}{\rho\left(\Phi_{0}\right)}\right)\right] \int_{-\infty}^{\zeta}\left(\frac{\partial \Phi_{0}}{\partial \zeta}\right)^{2} \mathrm{~d} \zeta
\end{aligned}
$$

and we have made use of Eq. (36b) for $P_{1}$. Eq. (42) may be manipulated to obtain the solvability condition

$$
0=\int_{-\infty}^{+\infty} \frac{1}{\rho\left(\Phi_{0}\right)} \frac{\partial \Phi_{0}}{\partial \zeta} R_{1} \mathrm{~d} \zeta
$$

which can be simplified to obtain the Clausius-Clapeyron relation

$$
0=-\Lambda \theta+\frac{\left(p_{\mathrm{S}}^{\star}-p^{\star}\right)}{\tilde{\gamma}}\left(1-\frac{\rho_{\mathrm{L}}}{\rho_{\mathrm{S}}}\right)+\left(\mathcal{K}_{1}+\mathcal{K}_{2}\right) \int_{-\infty}^{+\infty}\left(\frac{\partial \Phi_{0}}{\partial \zeta}\right)^{2} \mathrm{~d} \zeta
$$

This equation expresses temperature in terms of the bulk pressure and interface curvature. 
In dimensional form, the pressure jump condition (38) and the curvature-modified Clausius-Clapeyron relation (45) are

$$
\begin{aligned}
& \left.p\right|_{\mathrm{S}} ^{\mathrm{L}}=\gamma\left(\mathcal{K}_{1}+\mathcal{K}_{2}\right), \\
& 0=L\left(1-\frac{T}{T_{\mathrm{M}}}\right)+\left(p^{\mathrm{S}}-p_{\mathrm{R}}\right)\left(\frac{1}{\rho_{\mathrm{L}}}-\frac{1}{\rho_{\mathrm{S}}}\right)+\frac{\gamma}{\rho_{\mathrm{L}}}\left(\mathcal{K}_{1}+\mathcal{K}_{2}\right),
\end{aligned}
$$

where $\gamma$ is the surface tension (or excess Kramers potential) defined in Appendix B as

$$
\gamma=6 \gamma_{0} \int_{-\infty}^{+\infty}\left(\frac{\partial \Phi_{0}}{\partial \zeta}\right)^{2} \mathrm{~d} \zeta=6 \gamma_{0} \int_{0}^{1} \sqrt{\rho(\phi) H_{\mathrm{m}}(\phi)} \mathrm{d} \phi
$$

and $\Phi_{0}$ is determined by Eq. (41). Eqs. (46a) and (46b) determine the liquid pressure $p^{\mathrm{L}}$ and the temperature of the system $T$ in terms of the solid pressure $p^{\mathrm{S}}$, the interface curvature, and the material parameters.

The value of $\gamma$ is not in general equal to $\gamma_{0}$ defined by Eq. (11) but rather depends on the density ratio $\left(\rho_{\mathrm{S}} / \rho_{\mathrm{L}}\right)$ and the form of $\rho(\phi) H_{\mathrm{m}}(\phi)$. When $\rho(\phi) H_{\mathrm{m}}(\phi)=\phi^{2}(1-\phi)^{2}$ (so that the double-well potential is associated with a "per unit volume" quantity, see Eq. (7)) Eq. (41) admits the standard hyperbolic tangent profile and the integral in Eq. (47) evaluates to $\frac{1}{6}$, so that $\gamma=\gamma_{0}$. However, when $H_{\mathrm{m}}(\phi)=\phi^{2}(1-\phi)^{2}$ (so that the double-well potential is associated with a "per unit mass" quantity, see Eq. (7)) Eq. (41) no longer has the hyperbolic tangent profile solution. Additionally, with $r(\phi)=\phi^{2}(3-2 \phi)$ we find

$$
\gamma=\gamma_{0} \frac{2\left[\left(\rho_{\mathrm{S}} / \rho_{\mathrm{L}}\right)+\left(\rho_{\mathrm{S}} / \rho_{\mathrm{L}}\right)^{1 / 2}+1\right]}{3\left[\left(\rho_{\mathrm{S}} / \rho_{\mathrm{L}}\right)^{1 / 2}+1\right]},
$$

and with $r(\phi)=\phi$ we find

$$
\gamma=\gamma_{0} \frac{8\left[3+9\left(\rho_{\mathrm{S}} / \rho_{\mathrm{L}}\right)^{1 / 2}+11\left(\rho_{\mathrm{S}} / \rho_{\mathrm{L}}\right)+9\left(\rho_{\mathrm{S}} / \rho_{\mathrm{L}}\right)^{3 / 2}+3\left(\rho_{\mathrm{S}} / \rho_{\mathrm{L}}\right)^{2}\right]}{35\left[1+\left(\rho_{\mathrm{S}} / \rho_{\mathrm{L}}\right)^{1 / 2}\right]^{3}} .
$$

Each of the expressions for $\gamma$ in (48) and (49) evaluates to $\gamma_{0}$ when the bulk densities are equal. Furthermore, they are increasing functions of $\rho_{\mathrm{S}} / \rho_{\mathrm{L}}$ and vary approximately from $0.7 \gamma_{0}$ to $2.3 \gamma_{0}$ as $\rho_{\mathrm{S}} / \rho_{\mathrm{L}}$ varies from 0.1 to 10 .

This analysis shows that the equilibrium version of the present phase-field model allows an isothermal system in which the pressure difference between the two bulk phases is equal to the surface tension $\gamma$ times interface curvature (the Young-Laplace equation) while the temperature of the system is determined by $\gamma$ times interface curvature (the Gibbs-Thomson effect) and also by the bulk pressure, when there is a density mismatch, through a modified form of the Clausius-Clapeyron relation. The Clausius-Clapeyron pressure effect has been investigated by LaCombe et al. [34] as an additional control on the interface melting temperature during dendritic growth. We also note that Maruyama et al. [35] have examined transitions in the kinetic growth shapes of ice $I_{h}$, from a circular disk to a hexagonal plate, in response to pressure-induced alterations of the melting point near the roughening transition.

\section{Nonequilibrium: sharp-interface analysis}

In this section, we examine the role of nonequilibrium effects on the hydrodynamic boundary conditions that arise in the sharp-interface limit as well as the heat flux and Clausius-Clapeyron boundary conditions. 


\subsection{Scaling arguments}

In order to guide this analysis, we examine the Clausius-Clapeyron relation for a planar interface derived in AMW [1]. In terms of the present nondimensionalizations, their result is

$$
\begin{aligned}
-\epsilon \Lambda \theta-\frac{\epsilon}{\tilde{\gamma}}\left[\frac{\rho_{\mathrm{L}}}{\rho_{\mathrm{S}}}-1\right]\left(p_{\mathrm{S}}^{\star}-p^{\star}\right)= & \epsilon \tau \frac{\rho_{\mathrm{S}}}{\rho_{\mathrm{L}}} V_{n} \int_{-\infty}^{+\infty} \frac{1}{\left[\rho\left(\Phi_{0}\right)\right]^{2}}\left(\frac{\mathrm{d} \Phi_{0}}{\mathrm{~d} \zeta}\right)^{2} \mathrm{~d} \zeta+\frac{\epsilon}{\tilde{\gamma}} \frac{1}{2} V_{n}^{2}\left(\frac{\rho_{\mathrm{L}}}{\rho_{\mathrm{S}}}-1\right)^{2} \\
& +\frac{\operatorname{Pr}}{\tilde{\gamma}} \frac{\rho_{\mathrm{S}}}{\rho_{\mathrm{L}}} V_{n} \int_{-\infty}^{+\infty}\left[2 \mu\left(\Phi_{0}\right)+\lambda\left(\Phi_{0}\right)\right]\left[\frac{\mathrm{d}}{\mathrm{d} \zeta}\left(\frac{1}{\rho\left(\Phi_{0}\right)}\right)\right]^{2} \mathrm{~d} \zeta .
\end{aligned}
$$

The left-hand side of this equation contains the temperature and pressure terms. The right-hand side involves an interfacial attachment kinetics term, a volume-change term (which can also be interpreted as a velocity-dependent modification of the far field pressure owing to the vapor recoil effect [36]) and a viscous dissipation (or viscous drag) term, respectively. The attachment kinetics term is present in phase-field models without fluid flow [10] although the integral term here is modified by the density function. The viscous drag term is associated with the velocity change across the interfacial region in the normal direction and vanishes for inviscid flows or density-matched phases. In the analysis of solute trapping for a binary alloy by Ahmad et al. [37], an analogous effect, called solute drag, was identified (see the integral term in their Eq. (34)). In Eq. (50), the viscous drag term vanishes with the square of the density difference across the interface times the interface velocity while the solute drag effect in Ahmad et al. vanishes with the square of the concentration difference across the interface times the interface velocity.

In an asymptotic sense, it is possible to obtain a number of dominant balances among the physical effects represented by Eq. (50). In the case of equal densities and no flow possible balances include the situations where temperature is controlled at leading-order by attachment kinetics (e.g. if the interface is nearly planar), by curvature effects (the Gibbs-Thomson effect), by both effects, or by neither (e.g. see Refs. [10,23,27,28]). In the present context, we must determine how density differences and fluid flow affect the interface temperature. If in Eq. (50) all parameters are $\mathcal{O}(1)$ as $\epsilon \rightarrow 0$, then the viscous drag term dominates and no balance can be achieved. Hence we now reconsider the different terms in the equation in more detail.

Anderson et al. [1] found that for a typical material such as lead the viscous drag term was negligible compared to the attachment kinetics term due at least in part to a small density difference. This was true even for solid to liquid viscosity ratios of as large as $10^{6}$. Consequently, in Section 5.2, we consider an asymptotic regime in which the density difference $\left(\rho_{\mathrm{S}} / \rho_{\mathrm{L}}-1\right) \rightarrow 0$ as $\epsilon \rightarrow 0$.

In Section 5.3, we consider a second asymptotic regime in which the density difference is $\mathcal{O}(1)$ and the coefficient $\operatorname{Pr} / \tilde{\gamma}=C a / 6=\mathcal{O}(\epsilon)$ while the viscosity ratio $\mu_{\mathrm{S}} / \mu_{\mathrm{L}}$ is treated as $\mathcal{O}(1)$ with respect to $\epsilon$. This scaling may be more appropriate for two fluids (say liquid and vapor) where the density difference is not small and viscous effects are of secondary importance.

Another possible scaling would be to specify the viscosity function $\mu(\phi)$ to be $\mathcal{O}(\epsilon)$ in the interfacial region, where $\mathrm{d} \rho / \mathrm{d} \zeta$ is nonzero. However, it is not clear that this is physically plausible and we do not pursue this further.

As a simplification, in the remaining analyses we shall use exclusively the double-well potential given by $H_{\mathrm{v}}(\phi)=$ $\rho(\phi) H_{\mathrm{m}}(\phi)=\phi^{2}(1-\phi)^{2}$. We note, however, that in the case of nearly matched densities in Section $5.2, H_{\mathrm{v}}(\phi)$ and $H_{\mathrm{m}}(\phi)$ are equivalent at leading order.

\subsection{The case: $\rho_{\mathrm{S}} / \rho_{\mathrm{L}}-1=\mathcal{O}(\epsilon), \mathrm{Ca}=\mathcal{O}(1), \lambda=\mathcal{O}(\epsilon)$}

In this section, we describe a classical sharp-interface analysis where $\lambda=\epsilon \Lambda$ with $\Lambda=\mathcal{O}(1)$ as $\epsilon \rightarrow 0$ and we assume that the solid and liquid densities are nearly matched. In particular, we take 


$$
\rho(\phi)=1+\epsilon \chi r(\phi),
$$

where $\chi$ is $\mathcal{O}(1)$ as $\epsilon \rightarrow 0$.

We use the expansions

$$
\begin{aligned}
& \vec{u}\left(\zeta^{1}, \zeta^{2}, \zeta^{3}, t\right)=\vec{u}_{0}\left(\zeta^{1}, \zeta^{2}, \zeta^{3}, t\right)+\epsilon \vec{u}_{1}\left(\zeta^{1}, \zeta^{2}, \zeta^{3}, t\right)+\cdots \\
& p\left(\zeta^{1}, \zeta^{2}, \zeta^{3}, t\right)=p_{0}\left(\zeta^{1}, \zeta^{2}, \zeta^{3}, t\right)+\epsilon p_{1}\left(\zeta^{1}, \zeta^{2}, \zeta^{3}, t\right)+\cdots \\
& \phi\left(\zeta^{1}, \zeta^{2}, \zeta^{3}, t\right)=\phi_{0}\left(\zeta^{1}, \zeta^{2}, \zeta^{3}, t\right)+\epsilon \phi_{1}\left(\zeta^{1}, \zeta^{2}, \zeta^{3}, t\right)+\cdots \\
& \theta\left(\zeta^{1}, \zeta^{2}, \zeta^{3}, t\right)=\theta_{0}\left(\zeta^{1}, \zeta^{2}, \zeta^{3}, t\right)+\epsilon \theta_{1}\left(\zeta^{1}, \zeta^{2}, \zeta^{3}, t\right)+\cdots
\end{aligned}
$$

for the outer variables. The leading-order velocity and pressure in the bulk phases satisfy the incompressible Navier-Stokes equations

$$
\begin{aligned}
& \frac{\mathrm{D} \vec{u}_{0}}{\mathrm{D} t}=-\nabla p_{0}+\operatorname{Pr} \nabla \cdot \boldsymbol{\tau}_{0}, \\
& 0=\nabla \cdot \vec{u}_{0} .
\end{aligned}
$$

The phase-field equation gives $\phi_{0}=0$ in the liquid and $\phi_{0}=1$ in the solid and the energy equation becomes

$$
\frac{\mathrm{D} \theta_{0}}{\mathrm{D} t}=k \nabla^{2} \theta_{0}+\frac{S P r}{\Lambda \tilde{\gamma}} \tau_{0}: \nabla \vec{u}_{0},
$$

where $k=1$ in the liquid and $k=k_{\mathrm{S}} / k_{\mathrm{L}}$ in the solid.

The outer variables must satisfy interfacial conditions which we will determine by examining the inner problem and invoking matching and solvability conditions. In the inner region we put

$$
\begin{aligned}
& \vec{U}\left(\zeta^{1}, \zeta^{2}, \zeta, t\right)=\vec{U}_{0}\left(\zeta^{1}, \zeta^{2}, \zeta, t\right)+\epsilon \vec{U}_{1}\left(\zeta^{1}, \zeta^{2}, \zeta, t\right)+\cdots, \\
& P\left(\zeta^{1}, \zeta^{2}, \zeta, t\right)=\frac{1}{\epsilon}\left[P_{0}\left(\zeta^{1}, \zeta^{2}, \zeta, t\right)+\epsilon P_{1}\left(\zeta^{1}, \zeta^{2}, \zeta, t\right)+\cdots\right], \\
& \Phi\left(\zeta^{1}, \zeta^{2}, \zeta, t\right)=\Phi_{0}\left(\zeta^{1}, \zeta^{2}, \zeta, t\right)+\epsilon \Phi_{1}\left(\zeta^{1}, \zeta^{2}, \zeta, t\right)+\cdots, \\
& \Theta\left(\zeta^{1}, \zeta^{2}, \zeta, t\right)=\Theta_{0}\left(\zeta^{1}, \zeta^{2}, \zeta, t\right)+\epsilon \Theta_{1}\left(\zeta^{1}, \zeta^{2}, \zeta, t\right)+\cdots \\
& \boldsymbol{M}\left(\zeta^{1}, \zeta^{2}, \zeta, t\right)=\frac{1}{\epsilon}\left[\boldsymbol{M}_{0}\left(\zeta^{1}, \zeta^{2}, \zeta, t\right)+\epsilon \boldsymbol{M}_{1}\left(\zeta^{1}, \zeta^{2}, \zeta, t\right)+\cdots\right]
\end{aligned}
$$

We examine each of the governing equations in the following sections.

\subsubsection{Continuity equation}

The leading-order problem for the continuity equation (29) shows that $U_{0}^{3}$ is independent of $\zeta$. The matching condition for velocity is

$$
\lim _{\zeta \rightarrow \infty} \vec{U}_{0}=\left.\vec{u}_{0}\right|_{\mathrm{L}}, \quad \lim _{\zeta \rightarrow-\infty} \vec{U}_{0}=\left.\vec{u}_{0}\right|_{\mathrm{s}},
$$

where ' $\mathrm{L}_{\mathrm{L}}$ ' and ' $\mid \mathrm{s}$ ' denote a quantity in the bulk phase evaluated on the liquid and solid side of the interface, respectively. It follows that

$$
\left.\vec{u}_{0} \cdot \hat{n}\right|_{\mathrm{S}} ^{\mathrm{L}}=0 .
$$

This is the normal mass flux condition when the densities match to leading order. 


\subsubsection{Momentum equation}

At leading-order $\left[\mathcal{O}\left(\epsilon^{-1}\right)\right]$, the momentum equation (30) gives that

$$
\frac{\partial M_{0}^{31}}{\partial \zeta}=\frac{\partial M_{0}^{32}}{\partial \zeta}=\frac{\partial M_{0}^{33}}{\partial \zeta}=0
$$

Using (21a), (22a)-(22c) and the results in Appendix A we find that

$$
M_{0}^{31}=\operatorname{Pr} \tau_{0}^{31}, \quad M_{0}^{32}=\operatorname{Pr} \tau_{0}^{32}, \quad M_{0}^{33}=-\left[P_{0}+\frac{1}{2} \tilde{\gamma}\left(\frac{\partial \Phi_{0}}{\partial \zeta}\right)^{2}\right]+\operatorname{Pr} \tau_{0}^{33} .
$$

Integrating (58) once gives that these leading-order stresses are independent of $\zeta$. However, applying the matching condition on the stress gives

$$
\lim _{\zeta \rightarrow \pm \infty} \boldsymbol{M}_{0}=0
$$

and so $M_{0}^{31}=M_{0}^{32}=M_{0}^{33}=0$. Employing the expressions for $\tau_{0}^{31}, \tau_{0}^{32}$ and $\tau_{0}^{33}$ given in Appendix A we find

$$
\mu\left(\Phi_{0}\right) \frac{\partial U_{0}^{1}}{\partial \zeta}=0, \quad \mu\left(\Phi_{0}\right) \frac{\partial U_{0}^{2}}{\partial \zeta}=0, \quad-\left[P_{0}+\frac{1}{2} \tilde{\gamma}\left(\frac{\partial \Phi_{0}}{\partial \zeta}\right)^{2}\right]+\frac{4}{3} \operatorname{Pr} \mu\left(\Phi_{0}\right) \frac{\partial U_{0}^{3}}{\partial \zeta}=0,
$$

so that the forms of the velocity components and the pressure in the interfacial layer are

$$
\begin{aligned}
& U_{0}^{1}=b_{1}\left(\zeta^{1}, \zeta^{2}, t\right), \quad U_{0}^{2}=b_{2}\left(\zeta^{1}, \zeta^{2}, t\right), \quad U_{0}^{3}=b_{3}\left(\zeta^{1}, \zeta^{2}, t\right) \\
& P_{0}=-\frac{1}{2} \tilde{\gamma}\left(\frac{\partial \Phi_{0}}{\partial \zeta}\right)^{2},
\end{aligned}
$$

where $b_{1}, b_{2}$ and $b_{3}$ are independent of $\zeta$. The matching condition for velocity (56) then gives the no-slip condition

$$
\left.\left[\vec{u}_{0}-\hat{n}\left(\vec{u}_{0} \cdot \hat{n}\right)\right]\right|_{\mathrm{S}} ^{\mathrm{L}}=0,
$$

which shows that the tangential components of the leading-order velocities in the bulk phases are continuous across the interface.

Consistent with the leading-order inner stresses vanishing in the far-field limit, the leading-order inner pressure $P_{0}$ also vanishes in this limit. We emphasize that although the leading-order inner pressure term is $\mathcal{O}(1 / \epsilon)$ (see Eq. (55b)), the leading-order contribution to the stress is $\mathcal{O}(1)$ as $\boldsymbol{M}_{0}=0$. Stress jumps across the interface are determined next.

The momentum equation at $\mathcal{O}(1)$ in the inner region gives

$$
\begin{aligned}
& \left(U_{0}^{3}-V_{n}\right) \frac{\partial U_{0}^{1}}{\partial \zeta}=\frac{\partial M_{1}^{31}}{\partial \zeta}+\frac{\partial M_{0}^{11}}{\partial \zeta^{1}}+\frac{\partial M_{0}^{21}}{\partial \zeta^{2}}-\left(\mathcal{K}_{1}+\mathcal{K}_{2}\right) M_{0}^{31}-\mathcal{K}_{1}\left(M_{0}^{13}+M_{0}^{31}\right) \\
& \left(U_{0}^{3}-V_{n}\right) \frac{\partial U_{0}^{2}}{\partial \zeta}=\frac{\partial M_{1}^{32}}{\partial \zeta}+\frac{\partial M_{0}^{12}}{\partial \zeta^{1}}+\frac{\partial M_{0}^{22}}{\partial \zeta^{2}}-\left(\mathcal{K}_{1}+\mathcal{K}_{2}\right) M_{0}^{32}-\mathcal{K}_{1}\left(M_{0}^{23}+M_{0}^{32}\right) \\
& \left(U_{0}^{3}-V_{n}\right) \frac{\partial U_{0}^{3}}{\partial \zeta}=\frac{\partial M_{1}^{33}}{\partial \zeta}+\frac{\partial M_{0}^{13}}{\partial \zeta^{1}}+\frac{\partial M_{0}^{23}}{\partial \zeta^{2}}-\left(\mathcal{K}_{1}+\mathcal{K}_{2}\right) M_{0}^{33}+\mathcal{K}_{1} M_{0}^{11}+\mathcal{K}_{2} M_{0}^{22}
\end{aligned}
$$

However, using $M_{0}^{31}=M_{0}^{32}=M_{0}^{33}=0$, the symmetric nature of the stress tensor, Eqs. (62), and the expressions 
for the components of the stress tensor given in Appendix A, these equations simplify and we find that

$$
\begin{aligned}
& \frac{\partial M_{1}^{31}}{\partial \zeta}=-\frac{\partial}{\partial \zeta^{1}}\left[-P_{0}+\frac{1}{2} \tilde{\gamma}\left(\frac{\partial \Phi_{0}}{\partial \zeta}\right)^{2}\right], \\
& \frac{\partial M_{1}^{32}}{\partial \zeta}=-\frac{\partial}{\partial \zeta^{2}}\left[-P_{0}+\frac{1}{2} \tilde{\gamma}\left(\frac{\partial \Phi_{0}}{\partial \zeta}\right)^{2}\right], \\
& \frac{\partial M_{1}^{33}}{\partial \zeta}=-\left(\mathcal{K}_{1}+\mathcal{K}_{2}\right)\left[-P_{0}+\frac{1}{2} \tilde{\gamma}\left(\frac{\partial \Phi_{0}}{\partial \zeta}\right)^{2}\right] .
\end{aligned}
$$

Integrating these equations from $\zeta=-\infty$ to $\zeta=+\infty$ gives that

$$
\left.M_{1}^{31}\right|_{-\infty} ^{+\infty}=-\tilde{\gamma} \frac{\partial \mathcal{I}}{\partial \zeta^{1}},\left.\quad M_{1}^{32}\right|_{-\infty} ^{+\infty}=-\tilde{\gamma} \frac{\partial \mathcal{I}}{\partial \zeta^{2}},\left.\quad M_{1}^{33}\right|_{-\infty} ^{+\infty}=-\tilde{\gamma}\left(\mathcal{K}_{1}+\mathcal{K}_{2}\right) \mathcal{I}
$$

where, on using Eq. (63) the integral $\mathcal{I}$ may be expressed as

$$
\mathcal{I}=\int_{-\infty}^{+\infty}\left(\frac{\partial \Phi_{0}}{\partial \zeta}\right)^{2} \mathrm{~d} \zeta
$$

The integral $\mathcal{I}$ evaluates to $\frac{1}{6}$ for the double-well $H_{\mathrm{v}}(\phi)=\rho(\phi) H_{\mathrm{m}}(\phi)=\phi^{2}(1-\phi)^{2}$. Matching allows Eqs. (67) to be expressed in dimensional form as

$$
\begin{aligned}
& {\left.\left[\left(-p_{0} \boldsymbol{I}+\boldsymbol{\tau}_{0}\right) \cdot \vec{n}\right]_{\perp}\right|_{\mathrm{S}} ^{\mathrm{L}}=-\gamma_{0}\left(\mathcal{K}_{1}+\mathcal{K}_{2}\right),} \\
& {\left.\left[\boldsymbol{\tau}_{0} \cdot \vec{n}\right]_{\|}\right|_{\mathrm{S}} ^{\mathrm{L}}=0,}
\end{aligned}
$$

where the subscripts $\perp$ and $\|$ indicate components normal and parallel to the interface. Here $\boldsymbol{\tau}_{0}$ represents the leading-order outer viscous stress tensor. These are the conventional stress balances that hold in the absence of Marangoni effects, whereby the tangential stress is continuous across the interface and the normal stress difference across the interface is balanced by surface tension times interface curvature.

In the interest of modeling a solid-liquid system we examine the limit $\mu_{\mathrm{S}} / \mu_{\mathrm{L}} \rightarrow \infty$. In the bulk solid this limit gives at leading-order that $\nabla \cdot\left(\nabla \vec{u}_{\mathrm{S}}+\nabla \vec{u}_{\mathrm{S}}^{\mathrm{T}}\right)=0$ while the above stress boundary conditions give $\left[\left(\nabla \vec{u}_{\mathrm{S}}+\nabla \vec{u}_{\mathrm{S}}^{\mathrm{T}}\right) \cdot \hat{n}\right]_{\|}=$ 0 and $\left[\left(\nabla \vec{u}_{\mathrm{S}}+\nabla \vec{u}_{\mathrm{S}}^{\mathrm{T}}\right) \cdot \hat{n}\right]_{\perp}=0$. Hence the solid phase undergoes rigid body motion at leading order. The leading-order velocity field in the liquid phase satisfies the Navier-Stokes equations in the bulk and is subject to the normal flux and no-slip boundary conditions on the solid-liquid interface. As expected in this limit, the stress in the liquid is not imposed through a boundary condition but rather can be derived from the velocity field.

\subsubsection{Energy equation}

We next consider the energy equation (32) and recall that $v=\delta=(S / \epsilon \Lambda)=\mathcal{O}\left(\epsilon^{-1}\right)$. We note that $e=$ $\theta-r(\phi)+(S / 2 \Lambda \epsilon) H_{\mathrm{m}}(\phi)-\left(S p^{\star} / \Lambda \tilde{\gamma} \rho\right)$, so that in the inner region the advective term in the energy equation contributes at leading-order $\mathcal{O}\left(\epsilon^{-2}\right)$. The effects of dissipation as well as thermal diffusion also contribute at this order and we find that

$$
\frac{S}{2 \Lambda}\left(U_{0}^{3}-V_{n}\right) \frac{\partial H_{\mathrm{m}}}{\partial \zeta}=\frac{\partial}{\partial \zeta}\left[Q\left(\Phi_{0}\right) \frac{\partial \Theta_{0}}{\partial \zeta}\right]+\frac{S}{2 \Lambda}\left(U_{0}^{3}-V_{n}\right) \frac{\partial}{\partial \zeta}\left[\left(\frac{\partial \Phi_{0}}{\partial \zeta}\right)^{2}\right] .
$$

We note that the leading-order phase-field variable $\Phi_{0}$ satisfies Eq. (74), so that terms with coefficients $S / 2 \Lambda$ cancel. 
Eq. (70) can then be integrated twice to give

$$
\Theta_{0}=\int^{\zeta} \frac{d_{1}\left(\zeta^{1}, \zeta^{2}, t\right)}{Q\left(\Phi_{0}\right)} \mathrm{d} \zeta+d_{0}\left(\zeta^{1}, \zeta^{2}, t\right)
$$

where $d_{0}$ and $d_{1}$ are independent of $\zeta$. Matching the inner temperature to the outer solutions gives $d_{1}=0$, so that $\Theta_{0}=d_{0}\left(\zeta^{1}, \zeta^{2}, t\right)$ and $\left.\theta_{0}\right|_{\mathrm{L}}=\left.\theta_{0}\right|_{\mathrm{S}}=\Theta_{0}$. Therefore, the inner temperature $\Theta_{0}$ is independent of $\zeta$ and the outer temperature is continuous across the interface. In dimensional terms, we write $T_{\mathrm{L}}=T_{\mathrm{S}}=T_{\mathrm{I}}$, where $T_{\mathrm{I}}$ is the interface temperature as given in Eq. (78).

We can identify a heat flux boundary condition when we examine the $\mathcal{O}\left(\epsilon^{-1}\right)$ problem. The analysis involves the inner velocity component correction $U_{1}^{3}$, which is determined from the correction to the continuity equation. The details of this calculation are contained in Appendix C. We find that in dimensional form the heat flux boundary condition obtained is

$$
\left.k \hat{n} \cdot \nabla T\right|_{\mathrm{S}} ^{\mathrm{L}}=\left(\left.\vec{u}_{0}\right|_{\mathrm{L}} \cdot \hat{n}-V_{n}\right)\left[\rho_{\mathrm{L}} L+\gamma_{0}\left(\mathcal{K}_{1}+\mathcal{K}_{2}\right)\right] .
$$

This is the classical Stefan condition modified to account for motion in the solid and liquid bulk phases as well as the curvature of the interface. The effect of flow only enters in the first factor where it arises as the normal velocity of the material relative to the interface. The curvature effect follows from the term $\epsilon^{2} v \mathrm{D} \phi / \mathrm{D} t \nabla^{2} \phi$ in Eq. (32). It represents the internal energy gradient and double-well terms in our model. Similar curvature effects have been recognized by Umantsev and coworkers $[38,39]$ who identified the coefficient of the curvature to be $\gamma_{0}-T \partial \gamma_{0} / \partial T$. Eq. (72) is in agreement with this work when we observe from (11) that $\gamma_{0}$ is independent of temperature. Fife and Penrose [26] and Fried and Gurtin [40] have also identified this curvature effect in the context of phase-field models. In sharp-interface formulations Wollkind and Maurer [41], Umantsev and Davis [38], Zhang and Garimella [42] and Schlitz and Garimella [43] studied its effect on the stability of the interface and Lemieux and Kotliar [44] its influence on velocity selection during dendritic growth.

\subsubsection{Phase-field equation}

At leading-order, the phase-field equation (31) for nearly matched densities becomes

$$
0=\frac{\partial^{2} \Phi_{0}}{\partial \zeta^{2}}-\frac{1}{2} H_{\mathrm{m}}^{\prime}\left(\Phi_{0}\right)
$$

This equation has the first integral

$$
0=\left(\frac{\partial \Phi_{0}}{\partial \zeta}\right)^{2}-H_{\mathrm{m}}\left(\Phi_{0}\right)
$$

The first-order phase-field equation is given by

$$
\frac{\partial^{2} \Phi_{1}}{\partial \zeta^{2}}-\frac{1}{2} H_{\mathrm{m}}^{\prime \prime}\left(\Phi_{0}\right) \Phi_{1}=\tau\left(U_{0}^{3}-V_{n}\right) \frac{\partial \Phi_{0}}{\partial \zeta}+\left(\mathcal{K}_{1}+\mathcal{K}_{2}\right) \frac{\partial \Phi_{0}}{\partial \zeta}+\Lambda \Theta_{0} r^{\prime}\left(\Phi_{0}\right)+\frac{\chi}{2} \frac{\partial}{\partial \phi}\left[r\left(\Phi_{0}\right) H_{\mathrm{m}}\left(\Phi_{0}\right)\right],
$$

where we have used (63) and (74). Upon multiplying Eq. (75) by $\partial \Phi_{0} / \partial \zeta$ the solvability condition can be readily obtained

$$
\begin{aligned}
0= & \tau\left(U^{3}-V_{n}\right) \int_{-\infty}^{+\infty}\left(\frac{\partial \Phi_{0}}{\partial \zeta}\right)^{2} \mathrm{~d} \zeta+\left(\mathcal{K}_{1}+\mathcal{K}_{2}\right) \int_{-\infty}^{+\infty}\left(\frac{\partial \Phi_{0}}{\partial \zeta}\right)^{2} \mathrm{~d} \zeta+\Lambda \Theta_{0} \int_{-\infty}^{+\infty} \frac{\partial r\left(\Phi_{0}\right)}{\partial \zeta} \mathrm{d} \zeta \\
& +\frac{\chi}{2} \int_{-\infty}^{+\infty} \frac{\partial\left[r\left(\Phi_{0}\right) H_{\mathrm{m}}\left(\Phi_{0}\right)\right]}{\partial \zeta} \mathrm{d} \zeta .
\end{aligned}
$$


With $H_{\mathrm{m}}\left(\Phi_{0}\right)=\Phi_{0}^{2}\left(1-\Phi_{0}\right)^{2}$ and $r(0)=0$ and $r(1)=1$ we find that the interfacial condition is

$$
0=\tau\left(U_{0}^{3}-V_{n}\right)+\left(\mathcal{K}_{1}+\mathcal{K}_{2}\right)-6 \Lambda \Theta_{0},
$$

or in dimensional terms

$$
L\left(1-\frac{T_{\mathrm{I}}}{T_{\mathrm{M}}}\right)=-\frac{\gamma_{0}}{\rho_{\mathrm{L}}}\left(\mathcal{K}_{1}+\mathcal{K}_{2}\right)-\left(\left.\vec{u}_{0}\right|_{\mathrm{S}} \cdot \hat{n}-V_{n}\right) \frac{L}{T_{\mathrm{M}}} \frac{1}{\mu_{\mathrm{mob}}},
$$

where $T_{\mathrm{I}}$ is the interface temperature and $\mu_{\text {mob }}$ the interface mobility given by

$$
\mu_{\mathrm{mob}}=\frac{6 \rho_{\mathrm{L}} L l}{T_{\mathrm{M}} M} .
$$

Equation (78) gives the interface temperature $T_{\mathrm{I}}$ in terms of curvature and attachment kinetics and is equivalent (when $\vec{u}_{0} \mid \mathrm{s}=0$ ) to the result for no flow [10]. It differs from the more general Clausius-Clapeyron relation (see Eq. (102)) in that the effects of pressure, density change and viscous drag do not enter at leading order. We expect that this equation represents typical solid-liquid systems, in which the density difference between the two phases is small.

5.3. The case: $\mathrm{Ca}=\mathcal{O}(\epsilon), \rho_{\mathrm{S}} / \rho_{\mathrm{L}}-1=\mathcal{O}(1), \lambda=\mathcal{O}(\epsilon)$

As in the previous case, we conduct a classical sharp-interface analysis, but now we assume that the density difference is $\mathcal{O}(1)$ and the viscous effects are $\mathcal{O}(\epsilon)$. Specifically, we put $\operatorname{Pr} / \tilde{\gamma}=C a / 6=\mathcal{C} \epsilon$, where $\mathcal{C}=\mathcal{O}(1)$, and again take $\lambda=\Lambda \epsilon$, where $\Lambda=\mathcal{O}(1)$ as $\epsilon \rightarrow 0$.

We follow the same procedure as in the previous case and find that the leading-order outer problem remains the same with the exception that the Navier-Stokes equation (53a) is replaced by the Euler equation

$$
\rho \frac{\mathrm{D} \vec{u}_{0}}{\mathrm{D} t}=-\nabla p_{0}
$$

where in this equation $\rho=1$ in the liquid and $\rho=\rho_{\mathrm{S}} / \rho_{\mathrm{L}}$ in the solid and the energy equation is without the dissipation terms

$$
\rho \frac{\mathrm{D} \theta_{0}}{\mathrm{D} t}=k \nabla^{2} \theta_{0},
$$

where $k=1$ in the liquid and $k=k_{\mathrm{L}} / k_{\mathrm{S}}$ in the solid.

Interfacial conditions are derived by examining the inner region. Here we again use the expansions (55a)-(55e) and consider each equation in turn.

\subsubsection{Continuity equation}

The leading-order problem for the continuity equation (29) may be solved to obtain the form for $U_{0}^{3}$ through the interface as

$$
U_{0}^{3}=\frac{J_{0}\left(\zeta^{1}, \zeta^{2}, t\right)}{\rho\left(\Phi_{0}\right)}+V_{n}
$$

The matching condition (56) applied to the normal velocity component gives

$$
\left.\rho\left(\vec{u}_{0} \cdot \hat{n}-V_{n}\right)\right|_{\mathrm{S}} ^{\mathrm{L}}=0
$$

in dimensional form, where $J_{0}=\rho_{\mathrm{L}}\left(\left.\vec{u}_{0}\right|_{\mathrm{L}} \cdot \hat{n}-V_{n}\right)=\rho_{\mathrm{S}}\left(\left.\vec{u}_{0}\right|_{\mathrm{S}} \cdot \hat{n}-V_{n}\right)$ is identified as the mass flux across the interface. 


\subsubsection{Momentum equation}

At leading-order $\left[\mathcal{O}\left(\epsilon^{-1}\right)\right]$ the momentum equation (30) gives Eqs. (58) as in the analysis of Section 5.2. However, owing to the different scaling, Eqs. (21a), (22a)-(22c) and the results in Appendix A now lead to

$$
M_{0}^{31}=0, \quad M_{0}^{32}=0, \quad M_{0}^{33}=-\left[P_{0}+\frac{1}{2} \tilde{\gamma}\left(\frac{\partial \Phi_{0}}{\partial \zeta}\right)^{2}\right] .
$$

Eqs. (58) and the matching condition on the stress (60) require $M_{0}^{33}=0$, so that

$$
P_{0}=-\frac{1}{2} \tilde{\gamma}\left(\frac{\partial \Phi_{0}}{\partial \zeta}\right)^{2}
$$

The next order momentum equation in the inner region gives

$$
\begin{aligned}
& \rho\left(\Phi_{0}\right)\left(U_{0}^{3}-V_{n}\right) \frac{\partial U_{0}^{1}}{\partial \zeta}=\frac{\partial M_{1}^{31}}{\partial \zeta}+\frac{\partial M_{0}^{11}}{\partial \zeta^{1}}+\frac{\partial M_{0}^{21}}{\partial \zeta^{2}}-\left(\mathcal{K}_{1}+\mathcal{K}_{2}\right) M_{0}^{31}-\mathcal{K}_{1}\left(M_{0}^{13}+M_{0}^{31}\right), \\
& \rho\left(\Phi_{0}\right)\left(U_{0}^{3}-V_{n}\right) \frac{\partial U_{0}^{2}}{\partial \zeta}=\frac{\partial M_{1}^{32}}{\partial \zeta}+\frac{\partial M_{0}^{12}}{\partial \zeta^{1}}+\frac{\partial M_{0}^{22}}{\partial \zeta^{2}}-\left(\mathcal{K}_{1}+\mathcal{K}_{2}\right) M_{0}^{32}-\mathcal{K}_{1}\left(M_{0}^{23}+M_{0}^{32}\right), \\
& \rho\left(\Phi_{0}\right)\left(U_{0}^{3}-V_{n}\right) \frac{\partial U_{0}^{3}}{\partial \zeta}=\frac{\partial M_{1}^{33}}{\partial \zeta}+\frac{\partial M_{0}^{13}}{\partial \zeta^{1}}+\frac{\partial M_{0}^{23}}{\partial \zeta^{2}}-\left(\mathcal{K}_{1}+\mathcal{K}_{2}\right) M_{0}^{33}+\mathcal{K}_{1} M_{0}^{11}+\mathcal{K}_{2} M_{0}^{22} .
\end{aligned}
$$

However, using $M_{0}^{31}=M_{0}^{32}=M_{0}^{33}=0$, the symmetric nature of the stress tensor and the expressions in Appendix A with $\operatorname{Pr} / \tilde{\gamma}=\mathcal{C} \epsilon$ these equations simplify to give

$$
\begin{aligned}
& \frac{\partial M_{1}^{31}}{\partial \zeta}=J_{0} \frac{\partial U_{0}^{1}}{\partial \zeta}-\frac{\partial}{\partial \zeta^{1}}\left[-P_{0}+\frac{1}{2} \tilde{\gamma}\left(\frac{\partial \Phi_{0}}{\partial \zeta}\right)^{2}\right], \\
& \frac{\partial M_{1}^{32}}{\partial \zeta}=J_{0} \frac{\partial U_{0}^{2}}{\partial \zeta}-\frac{\partial}{\partial \zeta^{2}}\left[-P_{0}+\frac{1}{2} \tilde{\gamma}\left(\frac{\partial \Phi_{0}}{\partial \zeta}\right)^{2}\right], \\
& \frac{\partial M_{1}^{33}}{\partial \zeta}=J_{0} \frac{\partial U_{0}^{3}}{\partial \zeta}-\tilde{\gamma}\left(\mathcal{K}_{1}+\mathcal{K}_{2}\right)\left(\frac{\partial \Phi_{0}}{\partial \zeta}\right)^{2},
\end{aligned}
$$

where

$$
\begin{aligned}
& M_{1}^{31}=\tilde{\gamma}\left(-\frac{\partial \Phi_{0}}{\partial \zeta^{1}} \frac{\partial \Phi_{0}}{\partial \zeta}+\mathcal{C} \mu\left(\Phi_{0}\right) \frac{\partial U_{0}^{1}}{\partial \zeta}\right) \\
& M_{1}^{32}=\tilde{\gamma}\left(-\frac{\partial \Phi_{0}}{\partial \zeta^{2}} \frac{\partial \Phi_{0}}{\partial \zeta}+\mathcal{C} \mu\left(\Phi_{0}\right) \frac{\partial U_{0}^{2}}{\partial \zeta}\right) \\
& M_{1}^{33}=-P_{1}-p^{\star}+\tilde{\gamma}\left(-\frac{\partial \Phi_{0}}{\partial \zeta} \frac{\partial \Phi_{1}}{\partial \zeta}+\frac{4}{3} \mathcal{C} \mu\left(\Phi_{0}\right) \frac{\partial U_{0}^{3}}{\partial \zeta}\right)
\end{aligned}
$$

Now using these expressions and (85), Eqs. (87a)-(87c) become

$$
\tilde{\gamma} \frac{\partial}{\partial \zeta}\left[-\frac{\partial \Phi_{0}}{\partial \zeta^{1}} \frac{\partial \Phi_{0}}{\partial \zeta}+\mathcal{C} \mu\left(\Phi_{0}\right) \frac{\partial U_{0}^{1}}{\partial \zeta}\right]=J_{0} \frac{\partial U_{0}^{1}}{\partial \zeta}-\tilde{\gamma} \frac{\partial}{\partial \zeta^{1}}\left[\left(\frac{\partial \Phi_{0}}{\partial \zeta}\right)^{2}\right]
$$




$$
\begin{aligned}
& \tilde{\gamma} \frac{\partial}{\partial \zeta}\left[-\frac{\partial \Phi_{0}}{\partial \zeta^{2}} \frac{\partial \Phi_{0}}{\partial \zeta}+\mathcal{C} \mu\left(\Phi_{0}\right) \frac{\partial U_{0}^{2}}{\partial \zeta}\right]=J_{0} \frac{\partial U_{0}^{1}}{\partial \zeta}-\tilde{\gamma} \frac{\partial}{\partial \zeta^{2}}\left[\left(\frac{\partial \Phi_{0}}{\partial \zeta}\right)^{2}\right], \\
& \frac{\partial}{\partial \zeta}\left[-P_{1}-p^{\star}+\tilde{\gamma}\left(-\frac{\partial \Phi_{0}}{\partial \zeta} \frac{\partial \Phi_{1}}{\partial \zeta}+\frac{4}{3} \mathcal{C} \mu\left(\Phi_{0}\right) \frac{\partial U_{0}^{3}}{\partial \zeta}\right)\right]=J_{0} \frac{\partial U_{0}^{3}}{\partial \zeta}-\tilde{\gamma}\left(\mathcal{K}_{1}+\mathcal{K}_{2}\right)\left(\frac{\partial \Phi_{0}}{\partial \zeta}\right)^{2}
\end{aligned}
$$

The leading-order phase-field $\Phi_{0}$ satisfies the equilibrium equation (41) (see also Section 5.3.4) and depends only on $\zeta$. Consequently, we integrate Eqs. (89a) and (89b) to obtain

$$
\begin{aligned}
& U_{0}^{1}=b_{3}\left(\zeta^{1}, \zeta^{2}, t\right) \exp \left[\frac{J_{0}}{\tilde{\gamma} \mathcal{C}} \int^{\zeta} \frac{1}{\mu\left(\Phi_{0}\right)} \mathrm{d} \zeta\right]+b_{1}\left(\zeta^{1}, \zeta^{2}, t\right), \\
& U_{0}^{2}=b_{4}\left(\zeta^{1}, \zeta^{2}, t\right) \exp \left[\frac{J_{0}}{\tilde{\gamma} \mathcal{C}} \int^{\zeta} \frac{1}{\mu\left(\Phi_{0}\right)} \mathrm{d} \zeta\right]+b_{2}\left(\zeta^{1}, \zeta^{2}, t\right),
\end{aligned}
$$

where $b_{1}, b_{2}, b_{3}$ and $b_{4}$ are independent of $\zeta$. We now apply the matching condition for velocity (56) and find $b_{3}=b_{4}=0$, so that $U_{0}^{1}=b_{1}\left(\zeta^{1}, \zeta^{2}, t\right)$ and $U_{0}^{2}=b_{2}\left(\zeta^{1}, \zeta^{2}, t\right)$. Hence, the leading-order outer tangential velocity components are continuous across the interface

$$
\left.\left[\vec{u}_{0}-\hat{n}\left(\vec{u}_{0} \cdot \hat{n}\right)\right]\right|_{\mathrm{S}} ^{\mathrm{L}}=0 .
$$

These results give that $M_{1}^{31}=M_{1}^{32}=0$. However, integrating the momentum equation (89c) gives that $P_{1}$ is

$$
P_{1}=c_{3}\left(\zeta^{1}, \zeta^{2}, t\right)-p^{\star}-J_{0} U_{0}^{3}-\tilde{\gamma} \frac{\partial \Phi_{0}}{\partial \zeta} \frac{\partial \Phi_{1}}{\partial \zeta}+\tilde{\gamma}\left(\mathcal{K}_{1}+\mathcal{K}_{2}\right) \int_{-\infty}^{\zeta}\left(\frac{\partial \Phi_{0}}{\partial \zeta}\right)^{2} \mathrm{~d} \zeta+\frac{4}{3} \tilde{\gamma} \mathcal{C} \mu\left(\Phi_{0}\right) \frac{\partial U_{0}^{3}}{\partial \zeta}
$$

where $c_{3}$ is independent of $\zeta$. We next apply the matching principle for the pressure (37) to find that $c_{3}=\left.p_{0}\right|_{\mathrm{S}}+$ $p^{\star}+\left.J_{0} \vec{u}_{0}\right|_{\mathrm{S}} \cdot \vec{n}$ and the jump in the outer pressure at the interface is given (in dimensional form) by

$$
-\left.p\right|_{\mathrm{S}} ^{\mathrm{L}}=\frac{J_{0}^{2}}{\rho_{\mathrm{S}}}\left(\frac{\rho_{\mathrm{S}}}{\rho_{\mathrm{L}}}-1\right)-\gamma_{0}\left(\mathcal{K}_{1}+\mathcal{K}_{2}\right)
$$

where $J_{0}$ is given in the text after Eq. (83). Therefore, the pressure undergoes a jump across the interface due to the vapor recoil effect and interface curvature. Viscous stresses do not appear at this order in the analysis since $\mathrm{Ca}=\mathcal{O}(\epsilon)$.

\subsubsection{Energy equation}

We examine the energy equation (32) and find, as in the previous case, that $T_{\mathrm{L}}=T_{\mathrm{S}}=T_{\mathrm{I}}$; the outer temperature is continuous across the interface with the interface temperature $T_{\text {I }}$ given by Eq. (102). The heat flux boundary condition follows in an analogous manner as that of Section 5.2.3. The details of this calculation are given in Appendix D. We find that in dimensional form the heat flux boundary condition obtained is

$$
\left.k \hat{n} \cdot \nabla T\right|_{\mathrm{S}} ^{\mathrm{L}}=J_{0}\left\{L+\frac{\gamma_{0}\left(\mathcal{K}_{1}+\mathcal{K}_{2}\right)}{\rho_{\mathrm{L}}}+\left(\left.p\right|_{\mathrm{S}}-p_{\mathrm{R}}\right)\left[\frac{1}{\rho_{\mathrm{L}}}-\frac{1}{\rho_{\mathrm{S}}}\right]-\frac{J_{0}^{2}}{2}\left[\frac{1}{\rho_{\mathrm{L}}}-\frac{1}{\rho_{\mathrm{S}}}\right]^{2}\right\} .
$$

In addition to the latent heat and curvature terms on the left-hand side of this equation, which were also present in Eq. (72), we now have terms involving density jumps (e.g. see [36]). The last term on the right-hand side has the effect of reducing the effective latent heat, while the curvature term and pressure terms may contribute positively or negatively. We note that the terms on the right-hand side of this balance also appear in the modified Clausius-Clapeyron relation (102) and so can be related to the viscous dissipation and the interface kinetics effects. 


\subsubsection{Phase-field equation}

The leading-order phase-field equation is the same Eq. (41) as in the equilibrium analysis. An analysis of the phase-field equation may be pursued in a similar manner to that in the equilibrium case in Section 4.2 and the previous nonequilibrium case in Section 5.2.4. An alternate way [1] of deriving the modified Clausius-Clapeyron relation, however, is to examine the jump in Gibbs free energy $g$. One expression for $g$ follows from its definition in (20) and another follows directly from the phase-field equation (31). Equating these two expressions gives the Clausius-Clapeyron relation for a curved interface in nonequilibrium, as we show below.

Upon expanding the definition (20) as $g=g_{(0)}+\epsilon g_{(1)}$ (parentheses around the subscripts distinguish between the terms in the expansion and our previously defined $g_{0}$ in Eq. (7)) we find, using Eq. (85) that the jump in $g_{(0)}$ vanishes. At $\mathcal{O}(\epsilon)$, using Eqs. (85) and (92) and that $\left.\theta_{0}\right|_{\mathrm{S}} ^{\mathrm{L}}=0$, the definition of $g$ gives

$$
\left.g_{(1)}\right|_{\mathrm{S}} ^{\mathrm{L}}=-\Lambda \Theta_{0}+\frac{\left.p_{0}\right|_{\mathrm{S}}}{\tilde{\gamma}}\left[1-\frac{\rho_{\mathrm{L}}}{\rho_{\mathrm{S}}}\right]-\frac{J_{0}^{2}}{\tilde{\gamma}}\left(1-\frac{\rho_{\mathrm{L}}}{\rho_{\mathrm{S}}}\right)+\left(\mathcal{K}_{1}+\mathcal{K}_{2}\right) \int_{-\infty}^{\infty}\left(\frac{\partial \Phi_{0}}{\partial \zeta}\right)^{2} \mathrm{~d} \zeta,
$$

where we have used $r(0)=0$ and $r(1)=1$ but otherwise have not specified the form of $r(\phi)$.

We next examine the jump in $g$ as obtained directly from the phase-field equation (31). The leading-order phase-field equation can be written as

$$
\rho\left(\Phi_{0}\right) \frac{\partial g_{(0)}}{\partial \phi}=\frac{\partial^{2} \Phi_{0}}{\partial \zeta^{2}}
$$

Since $g=g(\theta, p, \phi)$ we have

$$
\frac{\partial g}{\partial \zeta}=\frac{\partial g}{\partial \theta} \frac{\partial \theta}{\partial \zeta}+\frac{\partial g}{\partial p} \frac{\partial p}{\partial \zeta}+\frac{\partial g}{\partial \phi} \frac{\partial \phi}{\partial \zeta} .
$$

Noting that $\Theta_{0}$ is independent of $\zeta$ and using Eqs. (85) and (96) we obtain

$$
\frac{\partial g_{(0)}}{\partial \zeta}=\frac{1}{\tilde{\gamma} \rho\left(\Phi_{0}\right)} \frac{\partial P_{0}}{\partial \zeta}+\frac{\partial g_{(0)}}{\partial \phi} \frac{\partial \Phi_{0}}{\partial \zeta}=\frac{1}{\rho\left(\Phi_{0}\right)} \frac{\partial}{\partial \zeta}\left[\frac{P_{0}}{\tilde{\gamma}}+\frac{1}{2}\left(\frac{\partial \Phi_{0}}{\partial \zeta}\right)^{2}\right]=0
$$

This gives $\left.g_{(0)}\right|_{S} ^{\mathrm{L}}=0$ which is consistent with the definition of $g$.

At the next order, the phase-field equation requires that $g_{(1)}$ satisfy

$$
\rho\left(\Phi_{0}\right) \frac{\partial g_{(1)}}{\partial \phi}=\frac{\partial^{2} \Phi_{1}}{\partial \zeta^{2}}-\left(\mathcal{K}_{1}+\mathcal{K}_{2}\right) \frac{\partial \Phi_{0}}{\partial \zeta}-\tau J_{0} \frac{1}{\rho\left(\Phi_{0}\right)} \frac{\partial \Phi_{0}}{\partial \zeta}-\left[\frac{1}{\rho\left(\Phi_{0}\right)} \frac{\partial \rho\left(\Phi_{0}\right)}{\partial \phi} \frac{\partial^{2} \Phi_{0}}{\partial \zeta^{2}}\right] \Phi_{1}
$$

We obtain from Eq. (97) at $\mathcal{O}(\epsilon)$ that

$$
\frac{\partial g_{(1)}}{\partial \zeta}=\frac{1}{\tilde{\gamma}} \frac{\partial}{\partial \phi}\left(\frac{1}{\rho\left(\Phi_{0}\right)}\right) \Phi_{1} \frac{\partial P_{0}}{\partial \zeta}+\frac{1}{\tilde{\gamma} \rho\left(\Phi_{0}\right)} \frac{\partial P_{1}}{\partial \zeta}+\frac{\partial g_{(1)}}{\partial \phi} \frac{\partial \Phi_{0}}{\partial \zeta}+\frac{1}{\rho\left(\Phi_{0}\right)} \frac{\partial^{2} \Phi_{0}}{\partial \zeta^{2}} \frac{\partial \Phi_{1}}{\partial \zeta},
$$

where we have used that $\Theta_{0}$ is independent of $\zeta$ and that $\partial g_{(0)} / \partial \theta=0$ (consistent with the dimensionless entropy in Eq. (19) having no contribution at $\mathcal{O}(1)$ when $\beta=0$ and $\lambda=\mathcal{O}(\epsilon)$ ). Some additional manipulations of (100) show that

$$
\begin{aligned}
\left.g_{(1)}\right|_{\mathrm{S}} ^{\mathrm{L}}= & -\tau J_{0} \int_{-\infty}^{+\infty} \frac{1}{\left[\rho\left(\Phi_{0}\right)\right]^{2}}\left(\frac{\partial \Phi_{0}}{\partial \zeta}\right)^{2} \mathrm{~d} \zeta-\frac{1}{2} \frac{J_{0}^{2}}{\tilde{\gamma}}\left[1-\left(\frac{\rho_{\mathrm{L}}}{\rho_{\mathrm{S}}}\right)^{2}\right] \\
& -\frac{4}{3} \mathcal{C} J_{0} \int_{-\infty}^{+\infty} \mu\left(\Phi_{0}\right)\left[\frac{\partial}{\partial \zeta}\left(\frac{1}{\rho\left(\Phi_{0}\right)}\right)\right]^{2} \mathrm{~d} \zeta .
\end{aligned}
$$


Now if we equate the two results (95) and (101) we find the modified Clausius-Clapeyron relation (in dimensional form) that includes nonequilibrium and curvature effects

$$
L\left(1-\frac{T_{\mathrm{I}}}{T_{\mathrm{M}}}\right)=\left(\left.p\right|_{\mathrm{S}}-p_{\mathrm{R}}\right)\left(\frac{1}{\rho_{\mathrm{S}}}-\frac{1}{\rho_{\mathrm{L}}}\right)-\frac{\gamma_{0}}{\rho_{\mathrm{L}}}\left(\mathcal{K}_{1}+\mathcal{K}_{2}\right)+\frac{J_{0}^{2}}{2}\left(\frac{1}{\rho_{\mathrm{S}}}-\frac{1}{\rho_{\mathrm{L}}}\right)^{2}-\frac{J_{0}}{\rho_{\mathrm{L}}}\left[\frac{M}{\rho_{\mathrm{L}}} \mathcal{I}_{1}+\frac{4}{3} \frac{\mu_{\mathrm{L}}}{l \rho_{\mathrm{L}}} \mathcal{I}_{2}\right],
$$

where

$$
\mathcal{I}_{1}=\int_{-\infty}^{+\infty} \frac{1}{\rho^{2}}\left(\frac{\partial \Phi_{0}}{\partial \zeta}\right)^{2} \mathrm{~d} \zeta, \quad \mathcal{I}_{2}=\int_{-\infty}^{+\infty} \mu\left(\Phi_{0}\right)\left[\frac{\partial}{\partial \zeta}\left(\frac{1}{\rho}\right)\right]^{2} \mathrm{~d} \zeta
$$

These integrals are evaluated for special cases in Appendix E.

Notice that the first three terms of Eq. (102) represent effects that are present in the equilibrium case while the remaining terms represent nonequilibrium effects associated with the change of phase. Also notice that $M / l \rho_{\mathrm{L}}=$ $6 L / T_{\mathrm{M}} \mu_{\mathrm{mob}}$ as defined in (79). As a consequence of our quasi-incompressibility assumption (see Eq. (6)) the effects of compressibility of the bulk fluid are not included in this equation. We refer the reader to [45], where the effects of isothermal compressibility have been included in a modified Clausius-Clapeyron relation for a solid-liquid system.

\section{Conclusions}

In this paper, we have examined sharp-interface limits of a phase-field model of solidification for a pure material that includes the effects of fluid motion. The model, given by Eqs. (29)-(32), was developed in Refs. [1,22]. Here we considered a simplified version that retains the square-gradient and double-well terms in the internal energy functional but excludes them in the entropy functional. The thermodynamically consistent derivation given in [1] and the identification of the distinguished limits which lead to useful free-boundary problems given in this paper together provide a validated phase-field model of solidification with convection. This paper gives the first sharp-interface analysis which addresses fluid flow and solidification in a phase-field model. However, we note that a similar sharp-interface calculation for a binary fluid (Cahn-Hilliard model) in the inviscid case has been carried out by Lowengrub and Truskinovsky [16].

The first important result in the present work follows from the sharp-interface analysis of the equilibrium version of the model in which we considered the classical limit $\lambda=\mathcal{O}(\epsilon)$ as $\epsilon \rightarrow 0$. Here the phase-field model leads to the Young-Laplace condition (46a) relating the jump in pressure between the bulk phases to the interface curvature. Also, a Clausius-Clapeyron condition (46b) relates the temperature of the system to the interface curvature and, when the bulk densities are different, the pressure as well. Therefore, even when there is no flow, a density difference between the bulk phases can affect the interfacial temperature.

In the first of two distinguished limits for the nonequilibrium case, we used $\lambda=\mathcal{O}(\epsilon), \rho_{\mathrm{S}} / \rho_{\mathrm{L}}-1=\mathcal{O}(\epsilon)$ with all other parameters treated as $\mathcal{O}(1)$, as $\epsilon \rightarrow 0$. The hydrodynamic boundary conditions of the associated free-boundary problem are the standard ones expected for a fluid-fluid interface undergoing phase transition (namely, the normal flux boundary condition (57), the condition of continuous tangential velocities (64) and the stress balance conditions (69a) and (69b)). It is important to point out that the condition of continuous tangential velocities and the stress balances both follow from the momentum equation. Viscosity is an essential element that ensures the continuity of tangential velocity because, in the sharp interface limit, the interface cannot support unbounded shear stresses and strains across it. Without viscosity we expect that the sharp-interface analysis would allow a discontinuity in the tangential velocity across the interface. The thermal interfacial conditions obtained in this case are also of the familiar types (namely, the continuity of the bulk temperatures at the interface, an expression for the interface 
temperature (78) and a condition involving the jump in heat flux across the interface (72)). However, we found that the heat flux boundary condition involves an interface curvature term proportional to surface energy $\gamma_{0}$. This term is often not included in standard free-boundary formulations. Its origin here is the source term in the energy equation (32) related to the gradient energy coefficient $\epsilon_{E}$ and is present with or without flow and density differences. The appearance of this term implies physically that the surface energy of the interface affects the balance of energy at the interface. Umantsev and Roitburd [39] found that the more general coefficient of the curvature term is $\gamma_{0}-T \mathrm{~d} \gamma_{0} / \mathrm{d} T$. We surmise that had we retained the square-gradient and double-well terms in the entropy functional we would have recovered this more general term in the sharp-interface limit. Finally, the interface temperature in this case is modified from its reference value $T_{\mathrm{M}}$ by interface curvature and attachment kinetics.

This limit allows for the interpretation of the solid as a highly viscous liquid if we take the subsequent limit $\mu_{\mathrm{S}} / \mu_{\mathrm{L}} \rightarrow \infty$. To leading-order in $\mu_{\mathrm{S}} / \mu_{\mathrm{L}}$ the corresponding free-boundary problem is satisfied by rigid body motion in the putative solid phase while the velocity in the liquid phase satisfies the Navier-Stokes equations subject to normal mass flux and no-slip conditions at the interface (see the discussion at the end of Section 5.2.2).

A second distinguished limit, given by $\lambda=\mathcal{O}(\epsilon)$ and $\operatorname{Pr} / \tilde{\gamma}=\mathcal{O}(\epsilon)$, is also possible in the nonequilibrium setting. This limiting case may be more appropriate for a fluid-fluid or fluid-vapor system where viscous effects may be of secondary importance. Here the hydrodynamic conditions are similar to the previous nonequilibrium case with the exception that the viscous terms are not present in the stress jump condition at leading-order (see Eqs. (83), (91) and (93)). The condition of continuous tangential velocities is still obtained, reflecting the limit of small but nonvanishing viscosity. The thermal interfacial conditions have the same general structure as those in the previous nonequilibrium case (see Eqs. (94) and (102)) but now include additional effects that can be attributed to density differences between the solid and liquid phases.

The phase-field model developed in [1] is the so-called generalized phase-field model, as it includes additional square-gradient and double-well contributions to both the internal energy and entropy functionals of the system. Similar models have been developed by other authors [14,33,40,46,47]. McFadden et al. [30] considered various sharp-interface limits of a generalized phase-field model and investigated the so-called thin interface limit, in which $\lambda=\mathcal{O}(1)$ and $\lambda \theta=\mathcal{O}(\epsilon)$ as $\epsilon \rightarrow 0$. They found that the generalized phase-field models can give rise to additional nonstandard terms in the thermal interfacial boundary conditions. Here we examined different asymptotic limits both with $\lambda=\mathcal{O}(\epsilon)$ as $\epsilon \rightarrow 0$ and also found that the leading-order thermal interfacial conditions involve new, albeit different, terms directly related to the square-gradient and double-well terms in the energy functional.

\section{Acknowledgements}

The authors would like to acknowledge support by the National Aeronautics and Space Administration Microgravity Science and Applications Program. We would also like to thank W.J. Boettinger for helpful conversations.

\section{Appendix A. Notes on differential geometry}

We employ body fitted coordinates $\left(\zeta^{1}, \zeta^{2}, \zeta^{3}\right)$ to the interface $\left[\phi=\frac{1}{2}\right.$ given by $\left.\vec{r}=\vec{r}_{0}\left(\zeta^{1}, \zeta^{2}, t\right)\right]($ e.g. see [48]). Here $\zeta^{1}, \zeta^{2}$ measure distance along the principal directions of the interface and $\zeta^{3}$ measures distance in the direction normal to the interface. Hence

$$
\vec{r}=\vec{r}_{0}\left(\zeta^{1}, \zeta^{2}, t\right)+\zeta^{3} \vec{n}
$$


where $\vec{n}$ is the unit normal to the the interface. The corresponding basis vectors are

$$
\vec{e}_{1}=\left(1-\zeta^{3} \mathcal{K}_{1}\right) \vec{t}_{1}, \quad \vec{e}_{2}=\left(1-\zeta^{3} \mathcal{K}_{2}\right) \vec{t}_{2}, \quad \vec{e}_{3}=\vec{n},
$$

where $\vec{t}_{1}, \vec{t}_{2}$ are the unit tangent vectors to the interface parallel to the principal directions. The corresponding metric tensor has nonzero components $g^{11}=\left(1-\zeta^{3} \mathcal{K}_{1}\right)^{-2}, g^{22}=\left(1-\zeta^{3} \mathcal{K}_{2}\right)^{-2}, g^{33}=1$.

The gradient of $\phi$ may be expressed as

$$
\nabla \phi=\frac{\partial \phi}{\partial \zeta^{1}} \vec{e}^{1}+\frac{\partial \phi}{\partial \zeta^{2}} \vec{e}^{2}+\frac{\partial \phi}{\partial \zeta^{3}} \vec{e}^{3}=\left[\left(1-\zeta^{3} \mathcal{K}_{1}\right)^{-2} \frac{\partial \phi}{\partial \zeta^{1}}\right] \vec{e}_{1}+\left[\left(1-\zeta^{3} \mathcal{K}_{2}\right)^{-2} \frac{\partial \phi}{\partial \zeta^{2}}\right] \vec{e}_{2}+\frac{\partial \phi}{\partial \zeta^{3}} \vec{e}_{3},
$$

where $\vec{e}^{k} \cdot \vec{e}_{j}=\delta_{j}^{k}$. Hence we find that

$$
|\nabla \phi|^{2}=\left(1-\zeta^{3} \mathcal{K}_{1}\right)^{-2}\left(\frac{\partial \phi}{\partial \zeta^{1}}\right)^{2}+\left(1-\zeta^{3} \mathcal{K}_{2}\right)^{-2}\left(\frac{\partial \phi}{\partial \zeta^{2}}\right)^{2}+\left(\frac{\partial \phi}{\partial \zeta^{3}}\right)^{2}
$$

The contravariant components of $\nabla \phi \otimes \nabla \phi$ are

$$
\left(\begin{array}{ccc}
{\left[\left(1-\zeta^{3} \mathcal{K}_{1}\right)^{-2} \frac{\partial \phi}{\partial \zeta^{1}}\right]^{2}} & \left(1-\zeta^{3} \mathcal{K}_{1}\right)^{-2}\left(1-\zeta^{3} \mathcal{K}_{2}\right)^{-2} \frac{\partial \phi}{\partial \zeta^{1}} \frac{\partial \phi}{\partial \zeta^{2}} & \left(1-\zeta^{3} \mathcal{K}_{1}\right)^{-2} \frac{\partial \phi}{\partial \zeta^{1}} \frac{\partial \phi}{\partial \zeta^{3}} \\
\left(1-\zeta^{3} \mathcal{K}_{1}\right)^{-2}\left(1-\zeta^{3} \mathcal{K}_{2}\right)^{-2} \frac{\partial \phi}{\partial \zeta^{1}} \frac{\partial \phi}{\partial \zeta^{2}} & {\left[\left(1-\zeta^{3} \mathcal{K}_{2}\right)^{-2} \frac{\partial \phi}{\partial \zeta^{2}}\right]^{2}} & \left(1-\zeta_{3} \mathcal{K}_{2}\right)^{-2} \frac{\partial \phi}{\partial \zeta^{2}} \frac{\partial \phi}{\partial \zeta^{3}} \\
\left(1-\zeta^{3} \mathcal{K}_{1}\right)^{-2} \frac{\partial \phi}{\partial \zeta^{1}} \frac{\partial \phi}{\partial \zeta^{3}} & \left(1-\zeta^{3} \mathcal{K}_{2}\right)^{-2} \frac{\partial \phi}{\partial \zeta^{2}} \frac{\partial \phi}{\partial \zeta^{3}} & {\left[\frac{\partial \phi}{\partial \zeta^{3}}\right]^{2}}
\end{array}\right) .
$$

We note that the contravariant components of the unit tensor in these coordinates is given by $I=\operatorname{diag}\left(g^{11}, g^{22}, g^{33}\right)$. Hence the expansion of $\boldsymbol{\sigma}^{\phi}=\tilde{\gamma} \epsilon\left[\frac{1}{2}|\nabla \phi|^{2} \boldsymbol{I}-\nabla \phi \otimes \nabla \phi\right]$ in the inner region (with $\zeta^{3}=\epsilon \zeta$ ) is $\boldsymbol{\sigma}^{\phi}=\epsilon^{-1}\left[\boldsymbol{\sigma}_{0}^{\phi}+\right.$ $\left.\epsilon \boldsymbol{\sigma}_{1}^{\phi}+\mathcal{O}\left(\epsilon^{2}\right)\right]$, where

$$
\boldsymbol{\sigma}_{0}^{\phi}=\tilde{\gamma}\left(\begin{array}{ccc}
\frac{1}{2}\left(\frac{\partial \Phi_{0}}{\partial \zeta}\right)^{2} & 0 & 0 \\
0 & \frac{1}{2}\left(\frac{\partial \Phi_{0}}{\partial \zeta}\right)^{2} & 0 \\
0 & 0 & -\frac{1}{2}\left(\frac{\partial \Phi_{0}}{\partial \zeta}\right)^{2}
\end{array}\right)
$$

and

$$
\boldsymbol{\sigma}_{1}^{\phi}=\tilde{\gamma}\left(\begin{array}{ccc}
\frac{\partial \Phi_{0}}{\partial \zeta} \frac{\partial \Phi_{1}}{\partial \zeta}+\zeta \mathcal{K}_{1}\left(\frac{\partial \Phi_{0}}{\partial \zeta}\right)^{2} & 0 & -\frac{\partial \Phi_{0}}{\partial \zeta^{1}} \frac{\partial \Phi_{0}}{\partial \zeta} \\
0 & \frac{\partial \Phi_{0}}{\partial \zeta} \frac{\partial \Phi_{1}}{\partial \zeta}+\zeta \mathcal{K}_{2}\left(\frac{\partial \Phi_{0}}{\partial \zeta}\right)^{2} & -\frac{\partial \Phi_{0}}{\partial \zeta^{2}} \frac{\partial \Phi_{0}}{\partial \zeta} \\
-\frac{\partial \Phi_{0}}{\partial \zeta^{1}} \frac{\partial \Phi_{0}}{\partial \zeta} & -\frac{\partial \Phi_{0}}{\partial \zeta^{2}} \frac{\partial \Phi_{0}}{\partial \zeta} & -\frac{\partial \Phi_{0}}{\partial \zeta} \frac{\partial \Phi_{1}}{\partial \zeta}
\end{array}\right) .
$$

In a similar way the expansion of $\boldsymbol{\sigma}^{p}=-\left(p+p^{\star}\right) \boldsymbol{I}$ in the inner region is given by $\boldsymbol{\sigma}^{p}=\epsilon^{-1}\left[\boldsymbol{\sigma}_{0}^{p}+\epsilon \boldsymbol{\sigma}_{1}^{p}+\mathcal{O}\left(\epsilon^{2}\right)\right]$, 
where the contravariant components of $\boldsymbol{\sigma}_{0}^{p}$ and $\boldsymbol{\sigma}_{1}^{p}$ are

$$
\boldsymbol{\sigma}_{0}^{p}=\left(\begin{array}{ccc}
-P_{0} & 0 & 0 \\
0 & -P_{0} & 0 \\
0 & 0 & -P_{0}
\end{array}\right)
$$

and

$$
\boldsymbol{\sigma}_{1}^{p}=\left(\begin{array}{ccc}
-P_{1}-p^{\star}-2 \zeta \mathcal{K}_{1} P_{0} & 0 & 0 \\
0 & -P_{1}-p^{\star}-2 \zeta \mathcal{K}_{2} P_{0} & 0 \\
0 & 0 & -P_{1}-p^{\star}
\end{array}\right)
$$

The viscous part of the stress tensor in the inner region is likewise given by $\boldsymbol{\tau}=\epsilon^{-1}\left[\boldsymbol{\tau}_{0}+\epsilon \boldsymbol{\tau}_{1}+\mathcal{O}\left(\epsilon^{2}\right)\right]$, where the contravariant components of $\boldsymbol{\tau}_{0}$ are

$$
\boldsymbol{\tau}_{0}=\left(\begin{array}{ccc}
-\frac{2}{3} \mu\left(\Phi_{0}\right) \frac{\partial U_{0}^{3}}{\partial \zeta} & 0 & \mu\left(\Phi_{0}\right) \frac{\partial U_{0}^{1}}{\partial \zeta} \\
0 & -\frac{2}{3} \mu\left(\Phi_{0}\right) \frac{\partial U_{0}^{3}}{\partial \zeta} & \mu\left(\Phi_{0}\right) \frac{\partial U_{0}^{2}}{\partial \zeta} \\
\mu\left(\Phi_{0}\right) \frac{\partial U_{0}^{1}}{\partial \zeta} & \mu\left(\Phi_{0}\right) \frac{\partial U_{0}^{2}}{\partial \zeta} & \frac{4}{3} \mu\left(\Phi_{0}\right) \frac{\partial U_{0}^{3}}{\partial \zeta}
\end{array}\right)
$$

The only component of $\boldsymbol{\tau}_{1}$ needed for the present calculations is $\tau_{1}^{33}$ which is given by

$$
\tau_{1}^{33}=\frac{4}{3}\left[\mu\left(\Phi_{0}\right) \frac{\partial U_{1}^{3}}{\partial \zeta}+\frac{\partial \mu}{\partial \phi} \Phi_{1} \frac{\partial U_{0}^{3}}{\partial \zeta}\right]-\frac{2}{3} \mu\left(\Phi_{0}\right)\left[\frac{\partial U_{0}^{1}}{\partial \zeta^{1}}+\frac{\partial U_{0}^{2}}{\partial \zeta^{2}}-\left(\mathcal{K}_{1}+\mathcal{K}_{2}\right) \frac{\partial}{\partial \zeta}\left(\zeta U_{0}^{3}\right)\right] .
$$

The components of $\boldsymbol{M}$ are then formed by

$$
\begin{aligned}
& \boldsymbol{M}_{0}=\boldsymbol{\sigma}_{0}^{p}+\boldsymbol{\sigma}_{0}^{\phi}+\operatorname{Pr} \boldsymbol{\tau}_{0}, \\
& \boldsymbol{M}_{1}=\boldsymbol{\sigma}_{1}^{p}+\boldsymbol{\sigma}_{1}^{\phi}+\operatorname{Pr} \boldsymbol{\tau}_{1} .
\end{aligned}
$$

We note that the components of the divergence of a second rank tensor $\boldsymbol{A}$ are given by

$$
A_{; i}^{i k}=\frac{1}{\sqrt{g}} \frac{\partial\left(\sqrt{g} A^{i k}\right)}{\partial \zeta^{i}}+A^{i j} \Gamma_{i j}^{k}
$$

where $g=g_{11} g_{22} g_{33}=\left(1-\zeta^{3} \mathcal{K}_{1}\right)^{2}\left(1-\zeta^{3} \mathcal{K}_{2}\right)^{2}$ and $\Gamma_{i j}^{k}$ are the so-called Christoffel symbols. We now expand $\nabla \cdot \boldsymbol{A}$ in the inner region. We note that the only Christoffel symbols with a nonzero order one contribution are

$$
\Gamma_{13}^{1}=\Gamma_{31}^{1}=-\mathcal{K}_{1}, \quad \Gamma_{23}^{2}=\Gamma_{32}^{2}=-\mathcal{K}_{2}, \quad \Gamma_{11}^{3}=\mathcal{K}_{1}, \quad \Gamma_{22}^{3}=\mathcal{K}_{2} .
$$

Hence if $\boldsymbol{A}=\boldsymbol{A}_{0}+\epsilon \boldsymbol{A}_{1}+\mathcal{O}\left(\epsilon^{2}\right)$ then

$$
\begin{aligned}
\nabla \cdot \boldsymbol{A}= & \frac{1}{\epsilon}\left[\frac{\partial A_{0}^{3 k}}{\partial \zeta}\right] \vec{e}_{k}+\left[\frac{\partial A_{1}^{3 k}}{\partial \zeta}+\frac{\partial A_{0}^{1 k}}{\partial \zeta^{1}}+\frac{\partial A_{0}^{2 k}}{\partial \zeta^{2}}-\left(\mathcal{K}_{1}+\mathcal{K}_{2}\right) A_{0}^{3 k}\right] \vec{e}_{k} \\
& -\mathcal{K}_{1}\left[A_{0}^{13}+A_{0}^{31}\right] \vec{e}_{1}-\mathcal{K}_{2}\left[A_{0}^{23}+A_{0}^{32}\right] \vec{e}_{2}+\left[A_{0}^{11} \mathcal{K}_{1}+A_{0}^{22} \mathcal{K}_{2}\right] \vec{e}_{3}+\mathcal{O}(\epsilon)
\end{aligned}
$$




\section{Appendix B. Surface energy}

The surface energy $\gamma$ may be defined as the excess Kramers potential (defined by $f-g$, where $f$ and $g$ are the Helmholtz and Gibbs free energies per unit mass) of an interface at the equilibrium temperature $T_{\mathrm{M}}$ at pressure $p_{\mathrm{R}}$. If we introduce a dividing surface at $z=z_{D}$ the surface energy is given by

$$
\begin{aligned}
& \gamma=\int_{-\infty}^{z_{D}}\left[\rho f-\rho g+\frac{\epsilon_{F}^{2}\left(T_{\mathrm{M}}\right)}{2} \phi_{z}^{2}-\rho_{\mathrm{S}} f_{\mathrm{S}}+\rho_{\mathrm{S}} g_{\mathrm{S}}\right] \mathrm{d} z+\int_{z_{D}}^{+\infty}\left[\rho f-\rho g+\frac{\epsilon_{F}^{2}\left(T_{\mathrm{M}}\right)}{2} \phi_{z}^{2}-\rho_{\mathrm{L}} f_{\mathrm{L}}+\rho_{\mathrm{L}} g_{\mathrm{L}}\right] \mathrm{d} z \\
& \gamma=\int_{-\infty}^{z_{D}}\left[-\left(p-p_{\mathrm{R}}\right)+\frac{\epsilon_{F}^{2}\left(T_{\mathrm{M}}\right)}{2} \phi_{z}^{2}\right] \mathrm{d} z+\int_{z_{D}}^{+\infty}\left[-\left(p-p_{\mathrm{R}}\right)+\frac{\epsilon_{F}^{2}\left(T_{\mathrm{M}}\right)}{2} \phi_{z}^{2}\right] \mathrm{d} z \\
& \gamma=\int_{-\infty}^{+\infty}\left[-\left(p-p_{\mathrm{R}}\right)+\frac{\epsilon_{F}^{2}\left(T_{\mathrm{M}}\right)}{2} \phi_{z}^{2}\right] \mathrm{d} z
\end{aligned}
$$

which is independent of the position of the dividing surface. In equilibrium, the stress in the interface is zero and hence $\boldsymbol{m}=0$ and thus from (36a) and (36b)

$$
p-p_{\mathrm{R}}+\frac{\epsilon_{F}^{2}\left(T_{\mathrm{M}}\right)}{2} \phi_{z}^{2}=0,
$$

and hence we may express the surface energy as

$$
\gamma=\int_{-\infty}^{+\infty} \epsilon_{F}^{2}\left(T_{\mathrm{M}}\right) \phi_{z}^{2} \mathrm{~d} z=6 \gamma_{0} \int_{-\infty}^{+\infty}\left(\frac{\partial \Phi_{0}}{\partial \zeta}\right)^{2} \mathrm{~d} \zeta
$$

where $\gamma_{0}=\frac{1}{6} \epsilon_{F}\left(T_{\mathrm{M}}\right) \sqrt{\rho_{\mathrm{L}} / 2 a}$ corresponds to the surface energy when $\rho_{\mathrm{S}}=\rho_{\mathrm{L}}$ (see Eq. (11)).

\section{Appendix C. Energy equation analysis: $\rho_{\mathrm{S}} / \rho_{\mathrm{L}}-1=\mathcal{O}(\epsilon)$}

Here we outline the calculation for the derivation in the sharp-interface limit of the heat flux boundary condition for the case $\lambda=\Lambda \epsilon$ and $\rho_{\mathrm{S}} / \rho_{\mathrm{L}}-1=\mathcal{O}(\epsilon)$ with all other parameters $\mathcal{O}(1)$ as $\epsilon \rightarrow 0$. In this case, we note that $\nu=\delta=S / \lambda=(S / \Lambda) \epsilon^{-1}$ and from Eq. (18) that $e=\mathcal{O}\left(\epsilon^{-1}\right)$.

At $\mathcal{O}\left(\epsilon^{-2}\right)$ the energy equation (32) gives that the leading-order temperature in the interfacial region is independent of $\zeta$ and hence may be expressed as $\Theta_{0}\left(\zeta^{1}, \zeta^{2}, t\right)$.

At the next order $\mathcal{O}\left(\epsilon^{-1}\right)$, we find that the energy equation gives that

$$
\begin{aligned}
\frac{\partial}{\partial \zeta}\left[Q\left(\Phi_{0}\right) \frac{\partial \Theta_{1}}{\partial \zeta}\right]= & \frac{S}{2 \Lambda}\left[\chi r\left(\Phi_{0}\right)\left(U_{0}^{3}-V_{n}\right)+\left(U_{1}^{3}-V_{n 1}\right)\right] \frac{\partial H_{\mathrm{m}}}{\partial \zeta} \\
& +\left(U_{0}^{3}-V_{n}\right) \frac{\partial}{\partial \zeta}\left[\Theta_{0}-r\left(\Phi_{0}\right)+\frac{S}{2 \Lambda} H_{\mathrm{m}}^{\prime}\left(\Phi_{0}\right) \Phi_{1}-\frac{S p^{\star}}{\Lambda \tilde{\gamma}}\right] \\
& -\frac{S}{\Lambda}\left\{\left(U_{0}^{3}-V_{n}\right) \frac{\partial}{\partial \zeta}\left(\frac{\partial \Phi_{0}}{\partial \zeta} \frac{\partial \Phi_{1}}{\partial \zeta}\right)+\frac{1}{2}\left(U_{1}^{3}-V_{n 1}\right) \frac{\partial}{\partial \zeta}\left[\left(\frac{\partial \Phi_{0}}{\partial \zeta}\right)^{2}\right]\right\} \\
& +\frac{S}{\Lambda}\left(\mathcal{K}_{1}+\mathcal{K}_{2}\right)\left(U_{0}^{3}-V_{n}\right)\left(\frac{\partial \Phi_{0}}{\partial \zeta}\right)^{2} \\
& +\frac{S}{\Lambda \tilde{\gamma}}\left[P_{0}\left(\frac{\partial U_{0}^{1}}{\partial \zeta^{1}}+\frac{\partial U_{0}^{2}}{\partial \zeta^{2}}+\frac{\partial U_{1}^{3}}{\partial \zeta}-\left(\mathcal{K}_{1}+\mathcal{K}_{2}\right) U_{0}^{3}\right)\right],
\end{aligned}
$$


and that the continuity equation gives

$$
\frac{\partial U_{1}^{3}}{\partial \zeta}+\frac{\partial U_{0}^{1}}{\partial \zeta^{1}}+\frac{\partial U_{0}^{2}}{\partial \zeta^{2}}-\left(\mathcal{K}_{1}+\mathcal{K}_{2}\right) U_{0}^{3}=-\chi\left(U_{0}^{3}-V_{n}\right) \frac{\partial r\left(\Phi_{0}\right)}{\partial \zeta} .
$$

Eq. (C.1) may be simplified by using (C.2), the leading-order phase-field equation (74), as well as the expression for $P_{0}$ given by (63) to yield

$$
\begin{aligned}
\frac{\partial}{\partial \zeta}\left[Q\left(\Phi_{0}\right) \frac{\partial \Theta_{1}}{\partial \zeta}\right]= & \frac{S}{2 \Lambda} \chi\left(U_{0}^{3}-V_{n}\right) \frac{\partial}{\partial \zeta}\left[r\left(\Phi_{0}\right) H_{\mathrm{m}}\left(\Phi_{0}\right)\right]-\frac{S}{\Lambda}\left(U_{0}^{3}-V_{n}\right) \frac{\partial}{\partial \zeta}\left(\frac{\partial \Phi_{0}}{\partial \zeta} \frac{\partial \Phi_{1}}{\partial \zeta}\right) \\
& +\left(U_{0}^{3}-V_{n}\right) \frac{\partial}{\partial \zeta}\left[\Theta_{0}-r\left(\Phi_{0}\right)+\frac{S}{2 \Lambda} H_{\mathrm{m}}^{\prime}\left(\Phi_{0}\right) \Phi_{1}-\frac{S p^{\star}}{\Lambda \tilde{\gamma}}\right] \\
& +\frac{S}{\Lambda}\left(U_{0}^{3}-V_{n}\right)\left(\mathcal{K}_{1}+\mathcal{K}_{2}\right)\left(\frac{\partial \Phi_{0}}{\partial \zeta}\right)^{2} .
\end{aligned}
$$

We integrate this once and apply the matching conditions

$$
\lim _{\zeta \rightarrow \infty} Q\left(\Phi_{0}\right) \frac{\partial \Theta_{1}}{\partial \zeta}=\left.\hat{n} \cdot \nabla \theta_{0}\right|_{\mathrm{L}}, \quad \lim _{\zeta \rightarrow-\infty} Q\left(\Phi_{0}\right) \frac{\partial \Theta_{1}}{\partial \zeta}=\left.\frac{k_{\mathrm{S}}}{k_{\mathrm{L}}} \hat{n} \cdot \nabla \theta_{0}\right|_{\mathrm{S}}
$$

to obtain

$$
\left.\hat{n} \cdot \nabla \theta_{0}\right|_{\mathrm{L}}-\left.\frac{k_{\mathrm{S}}}{k_{\mathrm{L}}} \hat{n} \cdot \nabla \theta_{0}\right|_{\mathrm{S}}=\left(u_{0}^{3}-V_{n}\right)\left[1+\frac{S}{\Lambda}\left(\mathcal{K}_{1}+\mathcal{K}_{2}\right) \int_{-\infty}^{+\infty}\left(\frac{\partial \Phi_{0}}{\partial \zeta}\right)^{2} \mathrm{~d} \zeta\right] .
$$

Noting that the integral in this expression evaluates to $\frac{1}{6}$, the dimensional form of this interface condition is

$$
k_{\mathrm{L}} \hat{n} \cdot \nabla T_{\mathrm{L}}-k_{\mathrm{S}} \hat{n} \cdot \nabla T_{\mathrm{S}}=\left(\left.\vec{u}_{0}\right|_{\mathrm{S}} \cdot \hat{n}-V_{n}\right)\left[\rho_{\mathrm{L}} L+\gamma_{0}\left(\mathcal{K}_{1}+\mathcal{K}_{2}\right)\right] .
$$

\section{Appendix D. Energy equation analysis: $\mathrm{Ca}=\mathcal{O}(\epsilon)$}

Here we outline the calculation for the derivation in the sharp-interface limit of the thermal boundary conditions for the case $\operatorname{Pr} / \tilde{\gamma}=\mathcal{C} \epsilon, \lambda=\Lambda \epsilon$ with all other parameters $\mathcal{O}(1)$ as $\epsilon \rightarrow 0$. As before, we note that $\nu=\delta=S / \lambda=$ $(S / \Lambda) \epsilon^{-1}$ and from Eq. (18) that $e=\mathcal{O}\left(\epsilon^{-1}\right)$.

As for the previous case with nearly matched densities at $\mathcal{O}\left(\epsilon^{-2}\right)$ the energy equation (32) gives that the leading-order temperature in the interfacial region is independent of $\zeta$ and hence may be expressed as $\Theta_{0}\left(\zeta^{1}, \zeta^{2}, t\right)$.

At next order $\mathcal{O}\left(\epsilon^{-1}\right)$ the energy equation (32) gives that

$$
\begin{aligned}
\frac{\partial}{\partial \zeta}\left[Q\left(\Phi_{0}\right) \frac{\partial \Theta_{1}}{\partial \zeta}\right]+\frac{S}{\Lambda} D_{1}= & \frac{S}{2 \Lambda} \rho\left(\Phi_{0}\right)\left[U_{1}^{3}-V_{n 1}-J_{0} \frac{\partial}{\partial \phi}\left(\frac{1}{\rho\left(\Phi_{0}\right)}\right) \Phi_{1}\right] \frac{\partial H_{\mathrm{m}}\left(\Phi_{0}\right)}{\partial \zeta} \\
& +J_{0} \frac{\partial}{\partial \zeta}\left[\Theta_{0}-r\left(\Phi_{0}\right)+\frac{S}{2 \Lambda} H_{\mathrm{m}}^{\prime}\left(\Phi_{0}\right) \Phi_{1}-\frac{S p^{\star}}{\Lambda \tilde{\gamma} \rho\left(\Phi_{0}\right)}\right],
\end{aligned}
$$

where $D_{1}$ represents the $\mathcal{O}\left(\epsilon^{-1}\right)$ contribution of the dissipation terms $\epsilon(S / \Lambda)(\mathrm{D} \Phi / \mathrm{D} t) \nabla^{2} \Phi+\mathcal{H}$ and is given by

$$
\begin{aligned}
D_{1}= & \left(U_{0}^{3}-V_{n}\right) \frac{\partial \Phi_{0}}{\partial \zeta}\left[\frac{\partial^{2} \Phi_{1}}{\partial \zeta^{2}}-\left(\mathcal{K}_{1}+\mathcal{K}_{2}\right) \frac{\partial \Phi_{0}}{\partial \zeta}\right]+\frac{\partial^{2} \Phi_{0}}{\partial \zeta^{2}}\left[\left(U_{1}^{3}-V_{n 1}\right) \frac{\partial \Phi_{0}}{\partial \zeta}+\left(U_{0}^{3}-V_{n}\right) \frac{\partial \Phi_{1}}{\partial \zeta}\right] \\
& +\left[-\frac{P_{1}+p^{\star}}{\tilde{\gamma}}+\frac{4}{3} \mathcal{C} \mu\left(\Phi_{0}\right) \frac{\partial U_{0}^{3}}{\partial \zeta}\right] \frac{\partial U_{0}^{3}}{\partial \zeta}-\frac{P_{0}}{\tilde{\gamma}}\left[\frac{\partial U_{1}^{3}}{\partial \zeta}+\frac{\partial U_{0}^{1}}{\partial \zeta^{1}}+\frac{\partial U_{0}^{2}}{\partial \zeta^{2}}-\left(\mathcal{K}_{1}+\mathcal{K}_{2}\right) U_{0}^{3}\right] .
\end{aligned}
$$


Here $P_{1}$ is given by Eq. (92) and $c_{3}\left(\zeta^{1}, \zeta^{2}, t\right)=p_{0}\left|\mathrm{~s}+p^{\star}+J_{0} \vec{u}_{0}\right|_{\mathrm{s}} \cdot \hat{n}$. The normal velocity correction $U_{1}^{3}$, which can be obtained by examining the continuity equation, is given by

$$
U_{1}^{3}-V_{n 1}=J_{0} \frac{\partial}{\partial \phi}\left(\frac{1}{\rho\left(\Phi_{0}\right)}\right) \Phi_{1}+\frac{1}{\rho\left(\Phi_{0}\right)}\left[b_{5}\left(\zeta^{1}, \zeta^{2}, t\right)+\int_{-\infty}^{\zeta} R \mathrm{~d} \zeta\right]
$$

where

$$
R=\left(\mathcal{K}_{1}+\mathcal{K}_{2}\right) \rho\left(\Phi_{0}\right) U_{0}^{3}-\rho\left(\Phi_{0}\right)\left(\frac{\partial U_{0}^{1}}{\partial \zeta^{1}}+\frac{\partial U_{0}^{2}}{\partial \zeta^{2}}\right)
$$

Using this result we can integrate Eq. (D.1) once and apply the matching conditions (C.4) to obtain

$$
\begin{aligned}
\left.\hat{n} \cdot \nabla \theta_{0}\right|_{\mathrm{L}}-\left.\frac{k_{\mathrm{S}}}{k_{\mathrm{L}}} \hat{n} \cdot \nabla \theta_{0}\right|_{\mathrm{S}}= & J_{0}-\frac{S p^{\star} J_{0}}{\Lambda \tilde{\gamma}}\left[1-\frac{\rho_{\mathrm{L}}}{\rho_{\mathrm{S}}}\right]-\frac{S}{\Lambda} \int_{-\infty}^{+\infty} D_{1} \mathrm{~d} \zeta \\
& +\frac{S}{2 \Lambda} \int_{-\infty}^{+\infty}\left[b_{5}\left(\zeta^{1}, \zeta^{2}, t\right)+\int_{-\infty}^{\zeta} R \mathrm{~d} \zeta\right] \frac{\partial H_{\mathrm{m}}}{\partial \zeta} \mathrm{d} \zeta .
\end{aligned}
$$

Next, we use the result for $U_{1}^{3}$ to simplify $D_{1}$ and find

$$
\begin{aligned}
D_{1}= & J_{0} \frac{\partial}{\partial \zeta}\left[\frac{1}{\rho\left(\Phi_{0}\right)} \frac{\partial \Phi_{0}}{\partial \zeta} \frac{\partial \Phi_{1}}{\partial \zeta}+\frac{1}{2}\left(\frac{\partial \Phi_{0}}{\partial \zeta}\right)^{2} \frac{\partial}{\partial \phi}\left(\frac{1}{\rho\left(\Phi_{0}\right)}\right) \Phi_{1}-\frac{\mathcal{K}_{1}+\mathcal{K}_{2}}{\rho\left(\Phi_{0}\right)} \int_{-\infty}^{\zeta}\left(\frac{\partial \Phi_{0}}{\partial \zeta}\right)^{2} \mathrm{~d} \zeta\right] \\
& +\frac{\partial}{\partial \zeta}\left[\frac{1}{2 \rho\left(\Phi_{0}\right)}\left(\frac{\partial \Phi_{0}}{\partial \zeta}\right)^{2}\left(b_{5}\left(\zeta^{1}, \zeta^{2}, t\right)+\int_{-\infty}^{\zeta} R \mathrm{~d} \zeta\right)\right] \\
& +\frac{1}{2}\left(\frac{\partial \Phi_{0}}{\partial \zeta}\right)^{2}\left(\frac{\partial U_{0}^{1}}{\partial \zeta^{1}}+\frac{\partial U_{0}^{2}}{\partial \zeta^{2}}-\left(\mathcal{K}_{1}+\mathcal{K}_{2}\right) U_{0}^{3}\right)+J_{0}\left[-\frac{p_{0} \mid \mathrm{s}+p^{\star}}{\tilde{\gamma}}+\frac{J_{0}}{\tilde{\gamma}}\left(U_{0}^{3}-\left.u_{0}^{3}\right|_{\mathrm{S}}\right)\right] \frac{\partial}{\partial \zeta}\left(\frac{1}{\rho\left(\Phi_{0}\right)}\right)
\end{aligned}
$$

If we note that $U_{0}^{3}-\left.u_{0}^{3}\right|_{\mathrm{S}}=J_{0} / \rho\left(\Phi_{0}\right)-\rho_{\mathrm{L}} J_{0} / \rho_{\mathrm{S}}$, it follows that

$$
\begin{aligned}
\int_{-\infty}^{+\infty} D_{1} \mathrm{~d} \zeta= & -J_{0}\left(\mathcal{K}_{1}+\mathcal{K}_{2}\right) \int_{-\infty}^{+\infty}\left(\frac{\partial \Phi_{0}}{\partial \zeta}\right)^{2} \mathrm{~d} \zeta+\frac{1}{2}\left(\frac{\partial U_{0}^{1}}{\partial \zeta^{1}}+\frac{\partial U_{0}^{2}}{\partial \zeta^{2}}\right) \int_{-\infty}^{+\infty}\left(\frac{\partial \Phi_{0}}{\partial \zeta}\right)^{2} \mathrm{~d} \zeta \\
& -\frac{1}{2}\left(\mathcal{K}_{1}+\mathcal{K}_{2}\right) \int_{-\infty}^{+\infty} U_{0}^{3}\left(\frac{\partial \Phi_{0}}{\partial \zeta}\right)^{2} \mathrm{~d} \zeta-\frac{J_{0}}{\tilde{\gamma}}\left(p_{0} \mid \mathrm{s}+p^{\star}\right)\left[1-\frac{\rho_{\mathrm{L}}}{\rho_{\mathrm{S}}}\right]+\frac{J_{0}^{3}}{2 \tilde{\gamma}}\left[1-\frac{\rho_{\mathrm{L}}}{\rho_{\mathrm{S}}}\right]^{2} .
\end{aligned}
$$

If we integrate the last term in Eq. (D.5) by parts, use Eqs. (D.4) and (D.7) and again note that $\left(\partial \Phi_{0} / \partial \zeta\right)^{2}=$ $\rho\left(\Phi_{0}\right) H_{\mathrm{m}}\left(\Phi_{0}\right)$ we find that

$$
\begin{aligned}
\hat{n} & \left.\cdot \nabla \theta_{0}\right|_{\mathrm{L}}-\left.\frac{k_{\mathrm{S}}}{k_{\mathrm{L}}} \hat{n} \cdot \nabla \theta_{0}\right|_{\mathrm{S}} \\
& =J_{0}\left\{1+\frac{S}{\Lambda}\left(\mathcal{K}_{1}+\mathcal{K}_{2}\right) \int_{-\infty}^{+\infty}\left(\frac{\partial \Phi_{0}}{\partial \zeta}\right)^{2} \mathrm{~d} \zeta+\left.\frac{S}{\Lambda \tilde{\gamma}} p_{0}\right|_{\mathrm{S}}\left[1-\frac{\rho_{\mathrm{L}}}{\rho_{\mathrm{S}}}\right]-\frac{S}{2 \Lambda \tilde{\gamma}} J_{0}^{2}\left[1-\frac{\rho_{\mathrm{L}}}{\rho_{\mathrm{S}}}\right]^{2}\right\} .
\end{aligned}
$$

In dimensional form this heat balance is

$$
k_{\mathrm{L}} \hat{n} \cdot \nabla T_{\mathrm{L}}-k_{\mathrm{S}} \hat{n} \cdot \nabla T_{\mathrm{S}}=J_{0}\left\{L+\frac{\gamma_{0}\left(\mathcal{K}_{1}+\mathcal{K}_{2}\right)}{\rho_{\mathrm{L}}}+\left(\left.p\right|_{\mathrm{S}}-p_{\mathrm{R}}\right)\left[\frac{1}{\rho_{\mathrm{L}}}-\frac{1}{\rho_{\mathrm{S}}}\right]-\frac{J_{0}^{2}}{2}\left[\frac{1}{\rho_{\mathrm{L}}}-\frac{1}{\rho_{\mathrm{S}}}\right]^{2}\right\} .
$$

Here we have assumed that $\rho\left(\Phi_{0}\right) H_{\mathrm{m}}\left(\Phi_{0}\right)=\Phi_{0}^{2}\left(1-\Phi_{0}\right)^{2}$, so that the integral in Eq. (D.8) evaluates to $\frac{1}{6}$. 


\section{Appendix E. Integrals in Clausius-Clapeyron relation}

Suppose $\rho(\phi) H_{\mathrm{m}}(\phi)=\phi^{2}(1-\phi)^{2}$ and $\rho(\phi)=1+\left(\rho_{\mathrm{S}} / \rho_{\mathrm{L}}-1\right) r(\phi)$. Then if $r(\phi)=\phi^{2}(3-2 \phi)$ we have

$$
\mathcal{I}_{1}=\int_{-\infty}^{+\infty} \frac{1}{\rho^{2}}\left(\frac{\partial \Phi_{0}}{\partial \zeta}\right)^{2} \mathrm{~d} \zeta=\frac{1}{6} \frac{\rho_{\mathrm{L}}}{\rho_{\mathrm{S}}}
$$

and if $r(\phi)=\phi$ with $s_{1} \equiv\left(\rho_{\mathrm{S}} / \rho_{\mathrm{L}}-1\right)$ we have

$$
\mathcal{I}_{1}=\int_{-\infty}^{+\infty} \frac{1}{\rho^{2}}\left(\frac{\partial \Phi_{0}}{\partial \zeta}\right)^{2} \mathrm{~d} \zeta=\frac{-2 s_{1}+\left(2+s_{1}\right) \ln \left(1+s_{1}\right)}{s_{1}^{3}}
$$

If $r(\phi)=\phi$ and $\mu(\phi)=1+s_{2} r(\phi)$, where $s_{2}=\left(\mu_{\mathrm{S}} / \mu_{\mathrm{L}}-1\right)$ then

$$
\mathcal{I}_{2}=\int_{-\infty}^{+\infty} \mu\left(\Phi_{0}\right)\left[\frac{\partial}{\partial \zeta}\left(\frac{1}{\rho}\right)\right]^{2} \mathrm{~d} \zeta=\frac{1}{6}\left[\frac{s_{1}^{2}}{\left(1+s_{1}\right)^{2}}+s_{2}\left(\frac{s_{1}\left(2 s_{1}^{2}+9 s_{1}+6\right)-6\left(1+s_{1}\right)^{2} \ln \left(1+s_{1}\right)}{s_{1}^{2}\left(1+s_{1}\right)^{2}}\right)\right] .
$$

If we fix $s_{2}, \mathcal{I}_{2}$ increases as $s_{1}$ increases or decreases away from zero. We note that for the expression in Eq. (E.3), $\mathcal{I}_{2} \sim \frac{1}{6} s_{1}^{2}\left(1+\frac{1}{2} s_{2}\right)$ when $s_{1} \ll 1$. A similar behavior is found for $\mathcal{I}_{2}$ with $r(\phi)=\phi^{2}(3-2 \phi)$.

\section{References}

[1] D.M. Anderson, G.B. McFadden, A.A. Wheeler, Physica D 135 (2000) 175-194.

[2] S.C. Huang, M.E. Glicksman, Acta Metall. 29 (1981) 717.

[3] M.E. Glicksman, S.C. Huang, Convective heat transfer during dendritic growth, in: J. Zierep, H. Oertel Jr. (Eds.), Convective Transport and Instability Phenomena, Braun, Karlsruhe, 1982, pp. 557-574.

[4] M.E. Glicksman, S.R. Coriell, G.B. McFadden, Ann. Rev. Fluid Mech. 18 (1986) 307.

[5] S.H. Davis, J. Fluid Mech. 212 (1990) 241.

[6] S.H. Davis, Effects of flow on morphological stability, in: D.T.J. Hurle (Ed.), Handbook of Crystal Growth, Vol. 1B, Elsevier, Amsterdam, 1993, pp. 859-897.

[7] G. Caginalp, in: L. Garrido (Ed.), Applications of Field Theory to Statistical Mechanics, Springer, Berlin, 1985, pp. 216-226.

[8] J.B. Collins, H. Levine, Phys. Rev. B 31 (1985) 6119.

[9] J.S. Langer, in: G. Grinstein, G. Mazenko (Eds.), Directions in Condensed Matter Physics, World Scientific, Philadelphia, PA, 1986, pp. $165-185$.

[10] S.-L. Wang, R.F. Sekerka, A.A. Wheeler, B.T. Murray, S.R. Coriell, R.J. Braun, G.B. McFadden, Physica D 69 (1993) 189.

[11] D.M. Anderson, G.B. McFadden, Phys. Fluids 9 (1997) 1870.

[12] D.M. Anderson, G.B. McFadden, A.A. Wheeler, Ann. Rev. Fluid Mech. 30 (1998) 139.

[13] O. Penrose, P.C. Fife, Physica D 43 (1990) 44.

[14] A.P. Umantsev, J. Chem. Phys. 96 (1992) 605.

[15] S.R. de Groot, P. Mazur, Non-equilibrium Thermodynamics, Dover, New York, 1984.

[16] J. Lowengrub, J. Truskinovsky, Proc. R. Soc. Ser. A 454 (1998) 2617.

[17] G. Caginalp, J. Jones, Appl. Math. Lett. 4 (1991) 97.

[18] G. Caginalp, J. Jones, in: M.E. Gurtin, G.B. McFadden (Eds.), On the Evolution of Phase Boundaries, The IMA Series in Mathematics and its Applications, Vol. 43, Springer, New York, 1992, pp. 27-50.

[19] H.J. Diepers, C. Beckermann, I. Steinbach, in: J. Beech, H. Jones (Eds.), Solidification Processing, Proceedings of the Fourth Decennial International Conference on Solidification Processing, University of Sheffield, Sheffield, 1997, pp. 426-430.

[20] R. Tönhardt, Convective effects on dendritic solidification, Ph. D. Thesis, Department of Mechanics, Royal Institute of Technology, Stockholm, Sweden, 1998.

[21] R. Tönhardt, G. Amberg, J. Cryst. Growth 194 (1998) 406.

[22] D.M. Anderson, G.B. McFadden, A.A. Wheeler, A phase-field model with convection: numerical simulations, in: M.K. Smith, et al. (Eds.), Interfaces for the 21st Century, Imperial College Press, London, 2001.

[23] G. Caginalp, Phys. Rev. A 39 (1989) 5887. 
[24] P.C. Fife, Dynamics of Internal Layers and Diffusive Interfaces, CBMS-NSF Regional Conference Series in Applied Mathematics, Vol. 53, SIAM, Philadelphia, PA, 1988.

[25] G.B. McFadden, A.A. Wheeler, R.J. Braun, S.R. Coriell, R.F. Sekerka, Phys. Rev. E 48 (1993) 2016.

[26] P.C. Fife, O. Penrose, Electron. J. Diff. Eqs. 1 (1995) 1.

[27] A. Karma, W.-J. Rappel, Phys. Rev. E 53 (1996) 3017.

[28] A. Karma, W.-J. Rappel, Phys. Rev. E 57 (1998) 4323.

[29] R.F. Almgren, SIAM J. Appl. Math. 59 (1999) 2086.

[30] G.B. McFadden, A.A. Wheeler, D.M. Anderson, Physica D 144 (2000) 154.

[31] A.A. Wheeler, G.B. McFadden, Eur. J. Appl. Math. 7 (1996) 367.

[32] A.A. Wheeler, G.B. McFadden, Proc. R. Soc. London A 453 (1997) 1611.

[33] C. Charach, P.C. Fife, Open Syst. Inform. Dyn. 5 (1998) 99.

[34] J.C. LaCombe, M.B. Koss, L.A. Tennenhouse, E.A. Winsa, M.E. Glicksman, J. Cryst. Growth 194 (1998) 143.

[35] M. Maruyama, Y. Kishimoto, T. Sawada, J. Cryst. Growth 172 (1997) 521.

[36] J.P. Burelbach, S.G. Bankoff, S.H. Davis, J. Fluid Mech. 195 (1988) 463.

[37] N.A. Ahmad, A.A. Wheeler, W.J. Boettinger, G.B. McFadden, Phys. Rev. E 58 (1998) 3436.

[38] A. Umantsev, S.H. Davis, Phys. Rev. A 45 (1992) 7195.

[39] A. Umantsev, A.L. Roitburd, Sov. Phys. 30 (1988) 651

[40] E. Fried, M.E. Gurtin, Physica D 91 (1996) 143.

[41] D.J. Wollkind, R.N. Maurer, J. Cryst. Growth 42 (1977) 24.

[42] L. Zhang, S. Garimella, J. Appl. Phys. 74 (1993) 2494.

[43] L.Z. Schlitz, S. Garimella, J. Appl. Phys. 76 (1994) 4863.

[44] M.-A. Lemieux, G. Kotliar, Phys. Rev. A 36 (1987) 4975.

[45] F. Baldoni, K.R. Rajagopal, Quart. Appl. Math. 55 (1997) 401.

[46] H.W. Alt, I. Pawlow, Physica D 59 (1992) 389.

[47] A.A. Wheeler, G.B. McFadden, W.J. Boettinger, Proc. R. Soc. London A 452 (1996) 495.

[48] R. Aris, Vectors, Tensors, and the Basic Equations of Fluid Mechanics, Dover, New York, 1962. 